\title{
La resistenza del giudicato alla violazione del principio di legalità penale
}

\author{
The resistance of res judicata to the \\ violation of the principle of penal legalty
}

A resistência da "coisa julgada" diante da violação do principio de legalidade penal

\section{Manfredi Bontempelfi ${ }^{1}$}

Università degli Studi di Milano/Italia manfredi.bontempelli@unimi.it http://lattes.cnpq.br/5307277119128221

(D) http://orcid.org/0000-0002-5735-8914

AвSTRAct: Va sottoposta ad attenta valutazione critica la tesi della giurisprudenza e di una parte della dottrina italiane, secondo cui il giudicato penale dovrebbe "cedere" di fronte alle violazioni dei diritti fondamentali accertate dalla Corte EDU, e delle garanzie costituzionali a seguito di interventi della Corte costituzionale. II presente scritto si sofferma sugli spazi di adeguamento del giudicato alla legalità penale davanti al giudice dell'esecuzione secondo il "diritto vivente", e sui relativi limiti in forza della legge processuale vigente. Le stesse eccezioni alla regola della forza esecutiva della sentenza irrevocabile, previste dal c.p.p. tassativamente, confermano I'intangibilità" del giudicato e rendono implausibile l'ipotesi di un suo "tramonto".

Parole Chiave: giudicato; esecuzione; diritti fondamentali; legge; giudice.

АвSTRACT: The thesis of the jurisprudence and of a part of the Italian doctrine, according to which the penal judgment should "surrender" in the face of violations of fundamental rights ascertained by EDU Court, and of constitutional guarantees

1 Professore Associato di Diritto processuale penale - Università degli Studi di Milano/Italia. 
as a result of interventions by the Constitutional Court, must be subjected to careful evaluation. The present paper focuses on the spaces for the adjudication of the sentence to the criminal legality before the judge of the execution according to the "living right", and on relative limits under the current procedural law. The same exceptions to the rule of enforceability of the irrevocable sentence, provided strictly by code of criminal procedure, confirm the "intangibility" of the judge and make implausible the hypothesis of his "sunset".

KeYwords: res iudicata, execution, fundamental rights, law, judge.

RESUMO: Deve-se submeter à atenta avaliação crítica a tese da jurisprudência e de uma parte da doutrina italiana segundo a qual a "coisa julgada" penal deveria "ceder" diante das violações dos direitos convencionais definidos pelo THDH, e das garantias constitucionais derivantes das intervenções da Corte Constitucional. O presente artigo analisa os espaços de conformidade do trânsito em julgado penal diante do juiz da execução segundo o "direito vivente" $^{\prime 2}$, e sobre seus relativos limites em razão da lei processual vivente. As mesmas exceções da força executiva da sentença irrevogável, previstas

2 N.d.T.: no sistema jurídico italiano, por "direito vivente" se entende o direito jurisprudencial consolidado, ou, mais em geral, o direito filtrado pela interpretação do juiz, pela opinião da doutrina, pela práxis administrativa e pelos comportamentos sociais. Dita expressão tem origens antigas: para alguns seria uma mera derivação do principio de efetividade, criado pelas canetas dos glossatori, passando através da doutrina estruturalistica e aquela institucional, e, por fim, utilizado até mesmo pela doutrina de direito civil e penal (ver: CARNELUTTI, Francesco. Giurisprudenza consolidata (ovvero della comodità del giudicare). Rivista di diritto processuale, 1949, I, p. 41). Em suas decisões, tanto para a Suprema Corte de Cassazione quanto para a Corte constitucional italiana, o formante fundamental do "direito vivente" seria a "consciência social", a "consciência comum", ou o "sentimento social", quais elementos que concorrem a realização do direito efetivo, que se diverge do direito escrito, ao qual é atribuído a tutela dos direitos subjetivos (entre as muitas decisões, a mero titulo exemplificativo, indicam-se: Corte constitucional, ordinanza de 15 de dezembro de 2005, numero 452, disponível em <http://www.giurcost.org>, acesso em: agosto de 2018. Dita locução assume particular relevância na época em que o direito escrito proveniente pelas fontes de uma pluralidade de ordenamentos as vezes colidentes e o direito jurisprudencial promana das concretas aplicações por parte de diferentes Cortes, nacionais e supranacionais, as quais se impõem continuamente em contrastes dialógicos entre si. Em dito contexto, a diferença entre direito escrito e direito efetivo se enriquece pela relevância atribuída às tradições constitucionais comuns e às raízes históricas e culturais dos diferentes ordenamentos. 
pelo c.p.p. taxativamente, confirmam a "intangibilidade" da "coisa julgada" e tornam implausível a hipótese de sua derrocada.

Palavras-chave: trânsito em julgado; execução; direitos fundamentais; lei; juiz.

SOMmario: 1. La progressiva "erosione" del giudicato penale, di fronte alla violazione dei diritti fondamentali accertata dalla Corte EDU. 2. La resistenza del giudicato, di fronte alla legalità penale riaffermata dalla Corte costituzionale. 3. II giudicato penale fra legge e "mito". 4. Spazi di adeguamento del giudicato alla legalità penale davanti al giudice dell'esecuzione, secondo il "diritto vivente". 5. L'intangibilità del giudicato nella giurisprudenza della Corte di cassazione. 6. La resistenza del giudicato all'overruling giurisprudenziale favorevole e il rispetto del nullum crimen.

\section{La progressiva "ERosione" del giudicato penale, di fronte ALLA VIOLAZIONE DEI DIRITTI FONDAMENTALI ACCERTATA DALLA Corte EDU}

Risulta assai problematica, e carica di notevoli conseguenze pratiche e sistematiche ${ }^{3}$, l'affermazione ripetuta ai più alti livelli della giurisprudenza italiana, secondo cui «il processo di erosione dell'intangibilità del giudicato [...] ha subito negli ultimi tempi una forte accelerazione, sotto la necessità di dare esecuzione all'obbligo di ripristinare i diritti del condannato, lesi da violazioni delle norme della Convenzione europea per la salvaguardia dei diritti dell'uomo e delle libertà fondamentali» ${ }^{4}$. È una

3 V., per considerazioni critiche di scenario sulla ricostruzione in discorso, CAPRIOLI, Francesco, Il giudice e la legge processuale: il paradigma rovesciato, in L'Indice penale, 2017, pp. 967 ss. e spec. pp. 973 ss. Cfr. anche, in termini generali, VIGONI, Daniela, Giudicato ed esecuzione penale: confini normativi e frontiere giurisprudenziali, in Processo penale e giustizia, 2015, n. 4, pp. 1 ss. Disponibile in www.processopenaleegiustizia.it. Ultimo accesso: 7 settembre 2018.

4 Cass., Sez. Un., 14 ottobre 2014, n. 42858, in C.E.D. Cass., rv. 260698, par. 8 motivazione. 
singolare concezione, quella in discorso, dal momento che vi è sottesa l'idea per cui il giudicato penale funzioni come fattore di compressione, e non di tutela, dei diritti fondamentali, il che stride con la fisionomia data all'istituto dalla legge processuale penale vigente.

La stessa scelta compiuta dal codice di rito, con l'art. 649, nel senso di caratterizzare il giudicato penale, a differenza di quello civile, attraverso la preclusione del secondo giudizio, con conseguente impossibilità di attribuire efficacia vincolante al contenuto di accertamento della decisione ${ }^{5}$, rende evidente il rapporto istituzionalmente sinergico - e non dialettico - fra il giudicato e i diritti fondamentali. Infatti, al ne bis in idem è assegnato il ben noto obiettivo di sottrarre il singolo «ad una teoricamente illimitata possibilità di persecuzione penale e, quindi, all'arbitrio incondizionato dell'organo punitivo» ${ }^{6}$, e non confliggono con questo prioritario scopo le «finalità di contenimento e razionalizzazione dei tempi e delle risorse processuali», che, secondo parte della dottrina, costituiscono la precipua ratio del divieto del secondo giudizio, quando esso «impedisce un possibile miglioramento della [...] situazione» giuridica «del soggetto già prosciolto o condannato con sentenza definitiva» ${ }^{7}$. Appare evidente, quindi, la posta in gioco quando si prospetta una "cedevolezza" del giudicato penale giustificata dallo scopo di attuare le garanzie sovranazionali. Il risultato cui conduce l'operazione in discorso non è necessariamente incoerente con il sistema processuale, se riguardante tassativi casi in cui sia necessario tutelare i diritti del condannato. Altro è trarre spunto dalla pretesa «flessibilizzazione» del giudicato ${ }^{8}$, per

5 Per questa lettura convincente, sostenuta da una parte autorevole della dottrina italiana, v., ad es., LOZZI, Gilberto, Preclusioni, II) Diritto processuale penale (voce), in Enciclopedia giuridica Treccani, vol. XXVI, Roma, 1991, p. 3, secondo cui «il giudicato penale è caratterizzato soltanto, per quanto concerne i rapporti con altri processi penali, da una mera efficacia preclusiva: quella del ne bis in idem».

6 DE LUCA, Giuseppe, I limiti soggettivi della cosa giudicata penale, Milano, Giuffrè, 1963, p. 90 nt. 34.

7 CAPRIOLI, Francesco, Il principio del ne bis in idem, in CAPRIOLI, Francesco; VICOLI, Daniele, Procedura penale dell'esecuzione, II ediz., Torino, Giappichelli, 2011, p. 73.

8 Di «flessibilizzazione» del giudicato parla espressamente, ad es., Cass., Sez. Un., 15 settembre 2015, n. 37107, in C.E.D. Cass., rv. 264859, par. 4 motivazione. V., in dottrina, ad es., TROISI, Paolo, Flessibilità del giudicato penale e 
sostenere che la sua intangibilità possa venir meno in generale, di fronte alla violazione delle garanzie CEDU (anche riconosciute a soggetti diversi dal condannato, ad esempio la vittima). Non c'è alcuna garanzia che ciò non conduca all'espunzione dal sistema della fondamentale ratio di tutela delle libertà del singolo, assegnata dalla legge al giudicato penale. Si delinea chiara, già a questo punto, l'esigenza di limitare a un numero chiuso di fattispecie previste dalla legge le vicende del possibile superamento degli effetti e dell'esecutività della decisione irrevocabile?

Come è noto, il problema della "resistenza" del giudicato penale alla suddetta "erosione" è emerso, nei tempi recenti, all'attenzione della giurisprudenza di legittimità e costituzionale interna, di fronte alle violazioni dei diritti fondamentali accertate dalla Corte EDU. È quanto accaduto nel noto "caso Ercolano", dove la Corte di cassazione, a Sezioni Unite, e la Corte costituzionale hanno riconosciuto in capo al giudice nazionale il dovere «di intervenire sul titolo esecutivo», appunto in deroga al giudicato, «per modificare la pena, quando la misura di questa è prevista da una norma di cui è stata riconosciuta l'illegittimità convenzionale», e di cui la stessa Consulta abbia dichiarato l'illegittimità costituzionale ${ }^{10}$. Nella specie, si trattava dell'art. 7 comma 1 d.1. n. 341/2000, nella parte in cui determinava l'applicazione retroattiva del più sfavorevole trattamento sanzionatorio, rispetto al precedente regime dell'art. 442 comma 2 c.p.p., nel caso di condanna all'ergastolo a seguito di giudizio abbreviato (vale a dire veniva applicata la pena dell'ergastolo, e non della reclusione di anni trenta, nel caso di determinazione della pena dell'ergastolo con

tutela dei diritti fondamentali, in Diritto penale contemporaneo, 2 aprile 2015. Disponibile in https://www.penalecontemporaneo.it/. Accesso: 7 settembre 2018. Si riferisce a una «(inevitabile) fragilità del giudicato nei casi di pena illegale», VICOLI, Daniele, L'illegittimità costituzionale della norma penale sanzionatoria travolge il giudicato: le nuove frontiere della fase esecutiva nei percorsi argomentativi delle Sezioni unite, in Rivista italiana di diritto e procedura penale, 2015, p. 1007.

9 Per la distinzione tra effetti ed esecuzione della sentenza penale irrevocabile v., in generale, LAVARINI, Barbara, L'esecutività della sentenza penale, Torino, Giappichelli, 2004, pp. 1 ss.

10 Così, Corte cost., 3 luglio 2013, n. 210, parr. 7.3 e 8 motivazione. V. poi Cass., Sez. Un., ord. 10 settembre 2012, n. 34472, in C.E.D. Cass., rv. 252934; Cass., Sez. Un., 7 maggio 2014, n. 18821, in C.E.D. Cass., rv. 258651. 
isolamento diurno). L'illegittimità costituzionale della norma è stata affermata dalla Consulta, per contrasto con l'art. 117 comma $1^{\circ}$ Cost., sulla scorta della sentenza della Grande Camera della Corte EDU, emessa il 17 settembre 2009 nel caso Scoppola c. Italia, per contrasto con l'art. 7 par. 1 Conv. EDU, ricomprendente il diritto dell'imputato di beneficiare della legge penale successiva alla commissione del reato che prevede una sanzione meno severa di quella stabilita in precedenza ${ }^{11}$. Dal canto suo, la Suprema Corte ha ritenuto che la citata applicazione retroattiva della disciplina in discorso producesse «attualmente ${ }^{12}$, essendo in esecuzione la pena dell'ergastolo inflitta al ricorrente, una permanente lesione dei diritti fondamentali di costui e l'ordinamento italiano», aggiungeva la Corte, «non può sottrarsi al dovere di rimuovere una simile situazione in forza dei principi affermati da Corte EDU, Scoppola c. Italia, verificando logicamente [...] la compatibilità con tali principi della normativa interna di riferimento» ${ }^{13}$.

Una violazione, dunque, "strutturale" del principio di legalità (di matrice convenzionale), da parte della norma sanzionatoria italiana, posta alla base della sua dichiarazione d'illegittimità costituzionale. Questo il presupposto del superamento del giudicato in situazioni, come quella al centro del "caso Ercolano", di mancata attivazione del rimedio in sede convenzionale (come avvenuto, con il ricorso al giudice di Strasburgo, nel

11 Infatti, come ricorda la stessa Corte di cassazione nel "caso Ercolano", sulla scia della giurisprudenza costituzionale, "profilandosi un contrasto tra una norma interna e una norma della CEDU, il giudice nazionale deve preventivamente verificare la praticabilità di una interpretazione della prima conforme alla norma convenzionale, ricorrendo a tutti i normali strumenti di ermeneutica giuridica»; dopodichè, in caso di «esito negativo di tale verifica», e di «contrasto non componibile in via interpretativa», è imposto «al giudice ordinario - che non può disapplicare la norma interna né farne applicazione, per il ritenuto contrasto con la CEDU e quindi con la Costituzione - di sottoporre alla Consulta la questione di legittimità costituzionale in riferimento all'art. 117, comma primo, Cost., [...], attraverso un rinvio pregiudiziale, con la conseguenza che l'eventuale operatività della norma convenzionale, così come interpretata dalla Corte di Strasburgo, deve passare attraverso una declaratoria d'incostituzionalità della normativa interna di riferimento o, se del caso, l'adozione di una sentenza interpretativa o additiva» (Cass., Sez. Un., ord. 10 settembre 2012, n. 34472, cit., par. 6 motivazione).

12 V., sul punto, infra, par. 5.

13 Cass., Sez. Un., ord. 10 settembre 2012, n. 34472, cit., par. 5 motivazione. 
citato "caso Scoppola"), nonostante l'identità della fattispecie rispetto a quella sindacata dalla Corte EDU. Secondo la Consulta in vicende simili, in cui «per l'ordinamento interno si è formato il giudicato», le «deroghe a tale limite vanno ricavate, non dalla CEDU, che non le esige, ma nell'ambito dell'ordinamento nazionale» ${ }^{14}$. Di qui l'interrogativo, peraltro non approfondito dalla giurisprudenza in discorso, sulla praticabilità di interpretazioni analogiche, con riguardo alle «ipotesi di flessione dell'intangibilità del giudicato, che la legge prevede nei casi in cui sul valore costituzionale ad esso intrinseco si debbano ritenere prevalenti opposti valori, ugualmente di dignità costituzionale, ai quali il legislatore intende assicurare un primato» ${ }^{15}$.

Sembra coerente attribuire alla legge (e non all'interprete) il compito di bilanciare i diversi interessi costituzionali e di definire gli spazi di tutela della libertà personale, a scapito della certezza del giudicato ${ }^{16}$, senza residui margini per un'interpretazione analogica ${ }^{17}$ che non parrebbe consentita alla luce del principio di tassatività delle deroghe alla forza esecutiva della sentenza irrevocabile ex art. 650 c.p.p., con conseguente

14 Corte cost., 3 luglio 2013, n. 210, par. 7.3 motivazione.

15 Corte cost., 3 luglio 2013, n. 210, par. 7.3 motivazione.

16 Diversamente, secondo la Corte di cassazione, «il valore della certezza del giudicato» dovrebbe «ritenersi recessivo rispetto ad evidenti e pregnanti compromissioni in atto di diritti fondamentali della persona. La preclusione, effetto proprio del giudicato, non può operare allorquando risulti pretermesso, con effetti negativi perduranti, un diritto fondamentale della persona, quale certamente è quello che incide sulla libertà: s'impone, pertanto, in questo caso di emendare "dallo stigma dell'ingiustizia" una tale situazione»: Cass., Sez. Un., ord. 10 settembre 2012, n. 34472, cit., par. 2 motivazione. Nello stesso senso, v. Cass., Sez. Un., 7 maggio 2014, n. 18821, cit., par. 7 motivazione, che prospetta la necessità di «un bilanciamento tra il valore costituzionale della intangibilità del giudicato e altri valori, pure costituzionalmente previsti, quale il diritto fondamentale e inviolabile alla libertà personale, la cui tutela deve ragionevolmente prevalere sul primo». In senso critico, CAPRIOLI, Francesco, Il giudice e la legge processuale, cit., p. 974.

17 V., invece, Cass., Sez. Un., 7 maggio 2014, n. 18821, cit., par. 7.3 motivazione, secondo cui all'ipotesi derogatoria del giudicato prevista dall'art. 2 comma $3^{\circ}$ c.p., «può essere accostato, in via analogica, il novum dettato dalla Corte EDU in tema di legalità convenzionale della pena, pur considerati i diversi effetti prodotti nell'ordinamento da una lex superveniens più favorevole rispetto a quelli derivanti da una sentenza di Strasburgo, alla quale consegua la declaratoria d'incostituzionalità della relativa norma interna» (corsivo aggiunto). 
«eccezionalità dei rimedi post iudicatum» ${ }^{18}$. Solo nei casi previsti dalla legge, e non in generale, come invece affermato dalla giurisprudenza, appare dunque fondato individuare spazi di permabilità del «giudicato sulla pena», «ad eventuali modifiche del trattamento sanzionatorio, purchè in bonam partem ${ }^{19}$. Inoltre, emerge l'esigenza di considerare il tema dei rapporti fra giudicato e diritti fondamentali a prescindere dalla sede in cui tali diritti vengono proclamati, e di non limitare il discorso relativo alla progressiva "erosione" del giudicato penale ai casi in cui siano in gioco $\mathrm{i}$ diritti del condannato riconosciuti a livello CEDU.

\section{LA RESISTENZA DEL GIUDICATO, DI FRONTE ALLA LEGALITÀ PENALE riaffermata dalla Corte costituzionale}

La stessa giurisprudenza della Suprema Corte sostiene la «valenza generale» della tesi sopra richiamata ${ }^{20}$, e la cedevolezza del giudicato penale di fronte «alle ipotesi in cui (senza alcun riferimento a sentenze della Corte EDU), per effetto di una intervenuta declaratoria di illegittimità costituzionale di una norma penale sostanziale, diversa da quella incriminatrice, sia ancora in atto una esecuzione di pena "illegittima" $»^{21}$. Secondo la pronuncia emessa dalla Corte di cassazione a Sezioni Unite nel "caso Gatto", «non esiste alcuna ragione per ritenere flessibile e cedevole il giudicato (quanto al trattamento sanzionatorio) fondato su norme nazionali violatrici della CEDU e, per contro, intangibile quello

18 V., ad es., LAVARINI, Barbara, I rimedi post iudicatum alla violazione dei canoni europei, in GAITO, Alfredo (a cura di), I princìpi europei del processo penale, Roma, Dike, 2016, p. 112.

19 Cass., Sez. Un., 15 settembre 2015, n. 37107, cit., par. 4 motivazione, secondo cui ciò sarebbe «espressione di un interesse collettivo, quello della certezza dei rapporti giuridici esauriti, suscettibile di bilanciamento con altri principi costituzionali e convenzionali, quali la libertà personale, la legalità della pena, la finalità rieducativa, il principio di uguaglianza, che, nella loro dimensione individuale, sono prevalenti rispetto alla dimensione collettiva sottesa all'esigenza di certezza dei rapporti giuridici».

20 V., adesivamente, CANZIO, Giovanni, La giurisdizione e la esecuzione della pena, in Diritto penale contemporaneo, 26 aprile 2016. Disponibile in https:// www.penalecontemporaneo.it/. Accesso: 7 settembre 2018.

${ }^{21}$ Cass., Sez. Un., 14 ottobre 2014, n. 42858, cit., par. 10.1 motivazione. 
fondato su norme dichiarate illegittime per violazione della Costituzione» ${ }^{22}$; in specie, per contrasto «con gli articoli 3 o 25, secondo comma, o 27, terzo comma, della Costituzione ovvero con tutte queste norme contemporaneamente» ${ }^{23}$.

È chiaro che quest'orientamento ha inteso coprire uno spazio di tutela lasciato aperto dai congegni tipizzati dalla legge, per derogare alla forza esecutiva della sentenza irrevocabile ex art. 650 c.p.p., nel caso in cui muti il quadro normativo di riferimento. La pronuncia d'incostituzionalità della norma penale sul trattamento sanzionatorio, per un verso, non consente di revocare la sentenza ai sensi dell'art. 673 c.p.p., secondo il meccanismo previsto per l'abrogazione o la dichiarazione d'illegittimità costituzionale della norma incriminatrice ${ }^{24}$. Per altro verso, non risulta invocabile il regime di applicazione retroattiva della legge sopravvenuta più favorevole, dettato dall'art. 2 comma $3^{\circ}$ c.p., in deroga alla regola generale di cui all'art. 2 comma $4^{\circ}$ c.p., per il caso di condanna a pena detentiva, cui sopravvenga una legge che prevede esclusivamente la pena pecuniaria ${ }^{25}$.

Inoltre, l'operatività dell'art. 30 comma $4^{\circ} 1$. n. 87/1953 non è scontata, ed anzi è da taluno contestata. Contro l'ipotesi estensiva deporrebbe, secondo una ricostruzione convincente, la chiara lettera della legge, nella parte in cui si riferisce alla cessazione di "tutti gli effetti penali",

22 Cass., Sez. Un., 14 ottobre 2014, n. 42858, cit., par. 10.1 motivazione. V., in dottrina, ad es., RUGGERI, Stefano, Giudicato costituzionale, processo penale, diritti della persona, in Diritto penale contemporaneo - Rivista trimestrale, 2015, n. 1, pp. 31 ss. Disponibile in https://www.penalecontemporaneo.it/. Accesso: 7 settembre 2018.

23 Cass., Sez. Un., 14 ottobre 2014, n. 42858, cit., par. 10.1 motivazione, con riferimento al caso del divieto di prevalenza della circostanza attenuante di cui all'art. 73, comma 5, d.P.R. 9 ottobre 1990, n. 309 (in tema di stupefacenti), sulla recidiva di cui all'art. 99, quarto comma, del codice penale, dichiarato illegittimo dalla sentenza n. 251 del 2012 della Corte costituzionale.

24 V., ad es., Cass., Sez. Un., ord. 10 settembre 2012, n. 34472, cit., par. 11 motivazione.

25 In tal caso, "la pena detentiva inflitta si converte immediatamente nella corrispondente pena pecuniaria, ai sensi dell'articolo 135" c.p. La previsione, introdotta dalla 1. n. 85/2006, fa eccezione alla regola generale dell'art. 2 comma $4^{\circ}$ c.p., in base alla quale la legge più favorevole si applica retroattivamente, "salvo che sia stata pronunciata sentenza irrevocabile". 
oltre che della "esecuzione" della sentenza irrevocabile di condanna ${ }^{26}$. Ciò, si è osservato, «implica necessariamente - alla evidenza - il radicale presupposto della abolitio criminis» ${ }^{27}$. Dal canto suo, la giurisprudenza dominante rigetta la tesi, ma con argomenti apodittici, o che comunque non colgono nel segno. Ha affermato, in particolare, un orientamento alla fine recepito dalle Sezioni Unite della Corte di cassazione ${ }^{28}$, che il citato art. 30 comma $4^{\circ}$ «non circoscrive in alcun modo, né direttamente né indirettamente, il divieto di dare esecuzione alla condanna pronunciata "in applicazione" di una norma penale dichiarata incostituzionale», e «si presta perciò ad essere letto nel senso di impedire anche solamente una parte dell'esecuzione, quella relativa alla porzione di pena che discendeva dall'applicazione della norma poi riconosciuta costituzionalmente illegittima $»^{29}$. Inoltre, secondo tale giurisprudenza, l'interpretazione

26 V., in particolare, GAMBARDELLA, Marco, Annullamento di circostanze aggravanti incostituzionali e revoca parziale del giudicato di condanna, in Cassazione penale, 2012 p. 1671; CAPRIOLI, Francesco, Giudicato e illegalità della pena: riflessioni a margine di una recente sentenza della Corte costituzionale, BARGIS, Marzia (a cura di), Studi in ricordo di Maria Gabriella Aimonetto, Milano, Giuffrè, 2013, p. 288.

27 Cass., Sez. I, 11 luglio 2012, n. 27640, in C.E.D. Cass., rv. 253383, par. 4.6 .6 motivazione, dove si è affermata l'«inconciliabilità del regime della cessazione di "tutti" gli effetti penali della condanna irrevocabile alla dichiarazione della illegittimità costituzionale di una mera circostanza aggravante del reato giudicato». A ciò Cass., Sez. Un., 7 maggio 2014, n. 18821, cit., par. 9.2 motivazione, ha obiettato «che il riferimento volutamente generico, contenuto nel richiamato art. 30, quinto comma, è certamente comprensivo» delle norme incriminatrici «(il che spiega il riferimento alla cessazione anche di "tutti" gli effetti penali), ma nulla induce a ritenere che sia circoscritto soltanto alle medesime».

Cass., Sez. Un., 7 maggio 2014, n. 18821, cit., par. 9.2 motivazione; Cass., Sez. Un., 15 settembre 2015, n. 37107, cit., par. 4 motivazione. V., in dottrina, VIGANÒ, Francesco, Pena illegittima e giudicato. Riflessioni in margine alla pronuncia delle Sezioni Unite che chiude la saga dei "fratelli minori" di Scoppola, in Diritto penale contemporaneo - Rivista trimestrale, 2014, n. 1. Disponibile in https://www.penalecontemporaneo.it/. Accesso: 7 settembre 2018, pp. 250 ss.

Cass., Sez. I, 13 gennaio 2012, n. 977, in C.E.D. Cass., rv. 252062, che ha ritenuto non consentita, appunto in forza degli artt. 136 Cost. e 30 commi $3^{\circ} \mathrm{e}$ $4^{\circ}$ 1. n. $87 / 1953$, l'esecuzione della porzione di pena inflitta dal giudice della cognizione in conseguenza dell'applicazione di una circostanza aggravante che sia stata successivamente dichiarata costituzionalmente illegittima. 
proposta sarebbe l'unica conforme «all'insieme dei principi costituzionali che regolano l'intervento repressivo penale e che impediscono di ritenere costituzionalmente giusta, e perciò eseguibile, anche soltanto una frazione della pena, se essa consegue all'applicazione di una norma contraria a Costituzione» ${ }^{30}$.

Il problema, tuttavia, non è se quella proposta sia l'unica soluzione esegetica compatibile con il quadro costituzionale di riferimento, e, «in particolare», con i «principi fissati dagli artt. 27, 3, 25, comma 2, Cost.» ${ }^{31}$, ma se quella stessa soluzione sia autorizzata dai dati della legge, in caso contrario emergendo, semmai, un problema di compatibilità costituzionale dell'art. 30 comma $4^{\circ}$ 1. n. 87/1953 (ovviamente risolvibile con l'intervento della Consulta, e non per via interpretativa). Non a caso, un'avveduta dottrina ha sostenuto che la Corte di cassazione, nella pronuncia delle Sezioni Unite relativa al "caso Ercolano", in cui ha sollevato la questione d'illegittimità costituzionale della norma censurata in sede europea nel "caso Scoppola", avrebbe dovuto contestare anche la legittimità costituzionale dell'art. 30 comma $4^{\circ}$, «nella parte in cui non prevede la caducazione del giudicato quando ad essere dichiarata illegittima sia una previsione normativa concernente il solo trattamento sanzionatorio» ${ }^{32}$.

Resterebbe, quindi, ancora da chiarire se il sistema processuale penale effettivamente attribuisca al giudicato un valore "cedevole", di fronte alla pur giusta esigenza di adattare l'esecuzione penale alla legalità riaffermata dalle pronunce della Corte costituzionale, o se, al contrario, la regola generale dell'“intangibilità" non debba esser ribadita, proprio in base alla constatazione che la legge prevede fattispecie derogatorie rispetto all'esecutività della sentenza irrevocabile (che non comprendono il caso della sopravvenuta declaratoria d'illegittimità costituzionale della norma

30 Cass., Sez. I, 13 gennaio 2012, n. 977, cit.

31 Così, anche, ad es., Cass., Sez. I, 9 luglio 2012, n. 26899, in C.E.D. Cass., rv. 253084.

32 CAPRIOLI, Francesco, Giudicato e illegalità della pena, cit., p. 289. Per la necessità di estendere la disciplina contenuta nell'art. 673 c.p.p. attraverso una sentenza additiva della Corte costituzionale o per via legislativa, v. GAMBARDELLA, Marco, Overruling favorevole della Corte europea e revoca del giudicato di condanna: a proposito dei casi analoghi alla sentenza "Scoppola", in Cassazione penale, 2012, p. 3999. 
sanzionatoria $)^{33}$. Per concludere che il giudicato debba "cedere" qualora sopravvenga un quadro normativo più favorevole (a seguito dell'intervento del giudice delle leggi), non è dunque sufficiente richiamare il fatto che l'esecuzione della pena prosegue (o addirittura ha avuto inizio) in violazione del principio di legalità penale. A rigore, di "esecuzione illegale" della pena potrebbe parlarsi solo in presenza di una fattispecie legale ad hoc, che preveda l'ineseguibilità della sentenza in presenza di specifici presupposti, non essendo sufficiente constatare che la pena viene eseguita ("illegittimamente") in violazione dei principi costituzionali. Andrebbero quindi distinti i concetti di "esecuzione illegale" della pena e di esecuzione della "pena illegittima".

\section{Il giudicato penale fra legge e "Mito"}

Rischia di provocare gravi equivoci la tendenza della giurisprudenza a contrapporre la «concezione tradizionale del giudicato», basata sulla regola dell'intangibilità, alla «proclamazione dei diritti fondamentali, che», secondo la Corte di cassazione, avrebbe «dato l'avvio ad una mutazione del fondamento e della stessa forza della cosa giudicata ${ }^{34}$. Si è affermato, in quest'ottica, che «la Costituzione e, successivamente, il nuovo codice di procedura penale hanno ridimensionato profondamente il significato totalizzante attribuito all'intangibilità del giudicato quale espressione della tradizionale concezione autoritaria dello Stato e ne hanno, per contro, rafforzato la valenza di garanzia individuale ${ }^{35}$. Tuttavia, non sembra condivisibile far risalire a una matrice autoritaria, e non democratica, la configurazione data al giudicato dal codice di rito del 1930, che a ben vede-

33 Al contrario, secondo Cass., Sez. Un., ord. 10 settembre 2012, n. 34472, cit., par. 5 motivazione, proprio l'art. 2 comma $3^{\circ}$ c.p. dimostrerebbe «la crisi dell'irrevocabilità del giudicato» ed a tale novità normativa potrebbe essere «accostato, in via analogica, il novum dettato dalla Corte EDU in tema di legalità della pena: in entrambi i casi, è l'esigenza imprescindibile di porre fine agli effetti negativi dell'esecuzione di una pena contra legem a prevalere sulla tenuta del giudicato, che deve cedere, anche in executivis, alla "più alta valenza fondativa dello statuto della pena”». V. anche, supra, nt. 17.

34 Cass., Sez. Un., 14 ottobre 2014, n. 42858, cit., par. 6.1 motivazione.

35 Cass., Sez. Un., 14 ottobre 2014, n. 42858, cit., par. 6.1 motivazione. 
re il codice vigente non ha innovato. Infatti, secondo la lettura preferibile, già il precedente regime rigettava la concezione, questa sì autoritaria, tesa a ravvisare nel giudicato un'efficacia di accertamento dell'illecito penale, vincolante gli altri giudizi penali, per abbracciare l'idea che il giudicato «si risolve», puramente e semplicemente, nel ne bis in idem ${ }^{36}$.

Una volta chiarito che ad essere in gioco non è la forza della cosa giudicata, identica secondo le codificazioni del 1930 e del 1988, ma il suo contenuto, ben circoscritto e pure esso costante nei due regimi, non residua spazio per ravvisare antitesi fra la «concezione tradizionale» della cosa giudicata e la tutela dei diritti fondamentali, affermatasi con il codice vigente, sulla scia dei valori costituzionali, in contrapposizione al codice previgente. Insomma, nel sistema odierno non c'è modo di porre in crisi l'idea del giudicato "intangibile", se correttamente intesa, considerando il mezzo attraverso il quale il giudicato stesso «si esplica» ${ }^{37}$ : è il ne bis in idem, in sede penale, a garantire l'incontrovertibilità del risultato del processo ${ }^{38}$, e ciò (punto importante ai presenti fini) risulta coerente alla natura della sentenza irrevocabile come «norma di origine giudiziaria» ${ }^{39}$. Come si è osservato in dottrina recentemente, «il divieto ex art. 649 c.p.p., in linea con la natura di norma del caso concreto propria della sentenza irrevocabile, mira a salvaguardare la stabilità e incontrovertibilità del tenore precettivo, cioè del "comando" contenuto nella sentenza» ${ }^{40}$.

36 Così, DE LUCA, Giuseppe, I limiti soggettivi della cosa giudicata penale, cit., p. 6 nt. 6: «Risolvendosi il giudicato nel puro e semplice ne bis in idem, ciò che rileva, ai fini del giudicato, non è tanto l'accertamento contenuto nella decisione, quanto il fatto della sentenza, ossia la sentenza come fatto giuridico»; LOZZI, Gilberto, Profili di una indagine sui rapporti tra «ne bis in idem» e concorso formale di reati, Milano, Giuffrè, 1974, p. 37: «la nota distintiva del giudicato penale è data esclusivamente dal ne bis in idem, vale a dire da un effetto giuridico ricollegabile alla mera irrevocabilità della sentenza». DE LUCA, Giuseppe, I limiti soggettivi, cit., p. 125. «L'indiscutibilità dell'accertamento è il risultato pratico del meccanismo attraverso cui opera il giudicato»: DE LUCA, Giuseppe, op. ult. cit., p. 134 nt. 35.

39 CORDERO, Franco, Procedura penale, VII ediz., Milano, Giuffrè, 1983, p. 1039.

40 RAFARACI, Tommaso, Ne bis in idem (voce), in Enciclopedia del diritto, Annali, vol. III, Milano, Giuffrè, 2010, p. 866 (corsivo aggiunto). 
Si ricava da questo dato un argomento a favore della ricostruzione dottrinale, cui ha aderito una parte minoritaria della giurisprudenza, secondo la quale l'“applicazione" della norma penale «si esaurisce» con la pronuncia della sentenza irrevocabile ${ }^{41}$, mentre la fase esecutiva «trova esclusivamente titolo nel relativo provvedimento di irrogazione della sanzione, il quale, in virtù della efficacia preclusiva del giudicato, è affatto insensibile a ogni questione circa la "applicazione" delle norme definitivamente operata dal giudice ${ }^{42}$. Senza possibilità, quindi, d'individuare momenti della fase esecutiva di possibile "applicazione" e delle norme incriminatrici e di quelle sanzionatorie. La tesi sarebbe fra l'altro confermata dallo stesso art. 30 comma $4^{\circ} 1$. n. 87/1953, nella parte in cui distingue il momento (passato) di applicazione della norma penale sostanziale ("quando è stata pronunciata sentenza di condanna in applicazione della norma dichiarata illegittima"), dal momento (presente) in cui vengono meno gli effetti del giudicato ("ne cessano l'esecuzione e gli effetti penali") ${ }^{43}$.

Semmai, il giudice dell'esecuzione applica le norme processuali che, nei casi e alle condizioni previste, consentono di revocare la sentenza di condanna (art. 673 c.p.p.) o di dichiarare il provvedimento mancante o non esecutivo (art. 670 c.p.p.). Pertanto, il tema sul tappeto è l'esistenza o no di «una norma in grado di sottrarre alla sentenza irrevocabile la forza esecutiva che l'art. 650 c.p.p. le attribuisce» ${ }^{44}$, in presenza di un certo «evento, a cui la legge attribuisce efficacia risolutiva del giudicato ${ }^{45}$, come accade nei casi di abrogazione della norma incriminatrice ex art. 2 comma $2^{\circ}$ c.p., di modifica del trattamento sanzionatorio ex art. 2 comma $3^{\circ}$ c.p. e di dichiarazione d'illegittimità costituzionale di una norma incriminatrice (o sanzionatoria, nell'interpretazione delle Sezioni Unite della Corte di cassazione) ex art. 30 comma $4^{\circ}$ 1. n. 87/1953. Se così non fosse, cioè se il giudice penale potesse riapplicare la disciplina sostanziale in sede esecutiva (dopo l'applicazione giudiziale in sede cognitiva), nell'ottica di verificare il

41 V., in tal senso, CAPRIOLI, Francesco, Giudicato e illegalità della pena, cit., pp. 270 ss.

42 Così, Cass., Sez. I, 13 gennaio 2012, n. 977, cit., par. 4.6.2 motivazione.

43 V., al riguardo, LAVARINI, Barbara, I rimedi post iudicatum alla violazione dei canoni europei, cit., p. 112.

44 V., in tal senso, CAPRIOLI, Francesco, op. ult. cit., p. 271.

45 LAVARINI, Barbara, op. loc. ult. cit. 
rispetto del principio di legalità ${ }^{46}$, sarebbe vanificata la stessa funzione del giudicato inteso come mezzo di concretizzazione della norma astratta ${ }^{47}$, con un ribaltamento del rapporto istituzionale fra la giurisdizione e la legge. Il che andrebbe a discapito di quella stessa tutela dei diritti fondamentali, che viene invocata, come si è visto anche dalla giurisprudenza, per propiziare un superamento del supposto «mito del giudicato».

Appare, dunque, scarsamente persuasiva la tesi della giurisprudenza di legittimità dominante, che (con inversione dei termini logici del problema) argomenta dalla ritenuta esistenza di un "obbligo di disapplicazione", il potere-dovere del giudice dell'esecuzione di incidere sul giudicato, rideterminando la pena inflitta sulla base di una norma penale sostanziale, poi dichiarata incostituzionale ${ }^{48}$. Con la conseguenza che la perdurante esecuzione della pena inflitta con la sentenza di condanna determinerebbe una «illegittima applicazione» di una norma di diritto penale sostanziale ${ }^{49}$. Invero, è proprio la «gerarchia delle fonti», che è stata invocata dalla medesima giurisprudenza a sostegno della tesi in esame ${ }^{50}$,

46 In questa direzione, v. Cass., Sez. Un., 7 maggio 2014, n. 18821, cit., par. 7 motivazione, che definisce «l'istanza di legalità della pena, per il vero», come «un tema che, in fase esecutiva, deve ritenersi costantemente sub iudice», senza essere «ostacolata dal dato formale della c.d. "situazione esaurita", che tale sostanzialmente non è, non potendosi tollerare che uno Stato democratico di diritto assista inerte all'esecuzione di pene non conformi alla CEDU e, quindi, alla Carta fondamentale».

47 Inoltre, il principio generale del ne bis in idem impone al giudice di applicare la legge sostanziale, concretizzandola, una sola volta. Sia consentito rinviare sul punto a BONTEMPELLI, Manfredi, La litispendenza penale, Milano, Giuffrè, 2017, pp. 88 ss. Cass., Sez. Un., 14 ottobre 2014, n. 42858, cit., par. 8.2 motivazione. Così, ad es., Cass., Sez. Un., 7 maggio 2014, n. 18821, cit., par. 7.1 motivazione, con riguardo agli «eventuali effetti ancora perduranti della violazione, determinata da una illegittima applicazione di una norma interna di diritto penale sostanziale interpretata in senso non convenzionalmente orientato». Addirittura, secondo questa pronuncia della Corte di cassazione, in tal caso la giurisdizione dovrebbe prendere «atto della insussistenza delle condizioni che giustificano l'esecuzione della pena nei confronti del condannato»e, quindi, «riportare la stessa in una dimensione di legittimità, utilizzando spazi di operatività della normativa vigente, che, benchè non chiaramente evidenziati, sono in essa implicati» (par. 7.2 motivazione).

50 Cass., Sez. Un., 14 ottobre 2014, n. 42858, cit., par. 8.2 motivazione, dove si esclude «che la sentenza che genera il comando punitivo del caso concreto si 
a subordinare l'intervento del giudice penale durante la fase esecutiva a una norma processuale che lo consenta.

È significativo come il «superamento culturale» cui si è fatto cenno venga sollecitato dalla giurisprudenza in discorso, richiamando «autorevoli voci della dottrina» della metà degli anni '50 del Novecento, «tese a evidenziare le distorsioni e le storture che il mito dell'intangibilità del giudicato determinava nella interpretazione di taluni istituti di diritto sostanziale» ${ }^{51}$. Giova ricordare, innanzitutto, che fra le situazioni in cui tale dottrina si domandava «perché, in sostanza, far prevalere sulla giustizia e sulla verità la intoccabile autorità del giudicato», veniva collocato, fra l'altro al primo posto, il «caso di sopravvenienza della prova di responsabilità dell'imputato assolto $»^{52}$. È l'ipotesi della revisione in peius, mai tramontata in alcuni ambienti culturali, che dimostra quali insidie contenga il "mito", questo sì tale, della "erosione del giudicato", per quanto riguarda la tutela dei diritti fondamentali. Suona dunque realistico, e non solo provocatorio, l'interrogativo che taluno ha posto di recente, di fronte al «nuovo sistema penale liquido»: «chi può escludere che un domani, venuto meno il "mito" del giudicato, esigenze di giustizia "sostanziale" e di uguaglianza non conducano alla soppressione del principio del ne bis in idem e del divieto di revisione in peius? ${ }^{23}$.

Inoltre, la dottrina che auspicava il superamento del «mito del giudicato», sottolineava il fondamento politico dell'istituto ${ }^{54}$, in campo

sottragga per questo solo alla gerarchia delle fonti e che gli effetti da essa prodotti possano resistere indenni alla declaratoria di illegittimità costituzionale della sanzione applicata».

51 Cass., Sez. Un., 14 ottobre 2014, n. 42858, cit., par. 6.1 motivazione. V. LEONE, Giovanni, Il mito del giudicato, in Scritti giuridici, vol. I, Napoli, Jovene, 1987, p. 63.

52 LEONE, Giovanni, op. loc. ult. cit.

53 MAZZA, Oliviero, Principio di legalità, diritto giurisprudenziale e giudice dell'esecuzione, in FIORIO, Carlo; FONTI, Rossella; MONTAGNA, Mariangela (a cura di), Inazione, controlli, esecuzione, Atti del Convegno in ricordo di Giovanni Dean, Pisa, Pacini Editore, 2017, p. 201.

54 «Fondamento politico e non logico, che, come esattamente avverte il Carnelutti non esiste, trattandosi solo di un problema di convenienza politica»: LEONE, Giovanni, Il mito del giudicato, cit., p. 69. Proprio perché di tipo politico, e non logico, si potrebbe proseguire, la scelta di attribuire la prevalenza al valore del giudicato rispetto ad altri valori deve essere riservata al legislatore e non all'interprete. 
penale a differenza che in quello civile, ricollegandosi alla «distinzione tra processo dispositivo e processo inquisitorio $»^{55}$, ed affermando che «il giudicato su rapporti indisponibili tanto guadagna in estensione quando perde in rigidità» ${ }^{56}$. Non sembra allora irragionevole riaffermare la rigidità del giudicato su una situazione indisponibile, quale è quella relativa al dovere di punire (oggetto di accertamento in sede penale), laddove il giudicato stesso venga circoscritto quanto ad estensione, come accade nel sistema vigente, refrattario all'idea della efficacia erga omnes dell'accertamento dei fatti su cui si basa la decisione irrevocabile ${ }^{57}$.

\section{Spazi di adeguamento del giudicato alla legalità penale DAVANTI AL GIUDICE DELL'ESECUZIONE, SECONDO IL "DIRITTO VIVENTE"}

Il quesito affrontato dalla Suprema Corte e dalla Consulta in ordine alla derogabilità del giudicato, nel caso di esecuzione di una pena "illegittima", perché inflitta in base a una norma dichiarata incostituzionale, interferisce con il quesito dell'istituto applicabile, per rimediare alla violazione del principio di legalità ${ }^{58}$. Infatti, il riconoscimento di spazi di adeguamento del giudicato agli interventi della Corte costituzionale sulla

55 LEONE, Giovanni, op. loc. ult. cit.

56 LEONE, Giovanni, op. cit., p. 70, in base all'osservazione che «il giudicato su rapporti indisponibili (tipico è il giudicato di stato) postula una efficacia universale che deriva dal fatto che la res iudicata si estende a tutta una larga ed indefinibile serie di relazioni che tale giudicato è chiamato a regolare; così tutta la società, nella quale tali relazioni si incardinano, è interessata alla validità sostanziale, alla rispondenza cioè a giustizia, della decisione».

57 Questo profilo è richiamato da Cass., Sez. Un., 14 ottobre 2014, n. 42858, cit., par. 6.3 motivazione. Non può pertanto esser condivisa nemmeno la tesi della «duplice dimensione del giudicato penale: la prima relativa all'accertamento del fatto, realmente intangibile [...]; la seconda relativa alla determinazione della pena, che, sprovvista di reale copertura costituzionale (o convenzionale), appare maggiormente permeabile alle "sollecitazioni" provenienti $a b$ extra rispetto alla res iudicata». Così, Cass., Sez. Un., 15 settembre 2015, n. 37107 , cit., par. 4 motivazione, che punta l'accento su una distinzione, del «giudicato sulla pena» dal «giudicato sull'accertamento», di cui non c'è traccia nel codice di rito vigente.

58 Per un'ampia sistematizzazione dall'angolo visuale dei rimedi, v. LAVARINI, Barbara, I rimedi post iudicatum alla violazione dei canoni europei, cit., pp. 87 ss. 
disciplina sanzionatoria, è andato di pari passo al riconoscimento di spazi sempre più estesi d'intervento in capo al giudice dell'esecuzione. La stessa possibilità di attivare il procedimento di esecuzione, per rideterminare la pena inflitta divenuta "illegittima", è stata posta al centro del giudizio di rilevanza della questione di legittimità costituzionale, sollevata nel già citato "caso Ercolano" 59 , alla luce del ravvisato «rapporto di strumentalità necessaria tra la risoluzione della questione di costituzionalità e la definizione dell'attivato incidente di esecuzione $»^{60}$.

Secondo la Suprema Corte, si è visto ${ }^{61}$, l'incidente di esecuzione verrebbe attivato in forza dell'art. 30 comma $4^{\circ}$ 1. n. 87 del 1953, disposizione che legittimerebbe «il superamento del giudicato di fronte alle primarie esigenze, insite nell'intero sistema penale, di tutelare il diritto fondamentale della persona alla legalità della pena anche in fase esecutiva e di assicurare parità di trattamento tra i condannati che versano in una identica situazione ${ }^{62}$. E si tratterebbe dell'incidente di esecuzione disciplinato dall'art. 670 c.p.p. ${ }^{63}$, istituto che, ritiene la medesima giurisprudenza, sarebbe impiegabile «per far valere tutte le questioni relative non solo alla mancanza o alla non esecutività del titolo, ma anche quelle che attengono alla eseguibilità e alla concreta attuazione del medesimo» ${ }^{64}$, ivi inclusi i vizi attinenti al «contenuto» del titolo esecutivo, che pertanto andrebbe sostituito, nelle situazioni qui discusse, «con un provvedimento dotato del requisito della eseguibilità» ${ }^{65}$. Questa prospettiva è stata condivisa dalla

59 Sul punto v., criticamente, CAPRIOLI, Francesco, Giudicato e illegalità della pena, cit., pp. 267 ss. Da una diversa prospettiva, LAMARQUE, Elisabetta, e VIGANOे, Francesco, Sulle ricadute interne della sentenza Scoppola, in Diritto penale contemporaneo, 31 marzo 2014. Così, Cass., Sez. Un., ord. 10 settembre 2012, n. 34472, cit., par. 11 motivazione.

${ }^{61}$ V., supra, par. 2.

${ }^{62}$ Cass., Sez. Un., ord. 10 settembre 2012, n. 34472, cit., par. 11 motivazione.

${ }^{63}$ Secondo la procedura dell'art. 666 c.p.p. V., ad es., Cass., Sez. Un., 15 settembre 2015, n. 37107, cit., par. 4 motivazione.

${ }^{64}$ Cass., Sez. Un., 7 maggio 2014, n. 18821, cit., par. 9.1 motivazione.

65 Cass., Sez. Un., 7 maggio 2014, n. 18821, cit., par. 9.1 motivazione. V., invece, Cass., Sez. Un., 15 settembre 2015, n. 37107, cit., par. 5.2 motivazione, che in relazione al «problema della illegalità sopravvenuta della pena nella sentenza di patteggiamento irrevocabile», esclude il ricorso al rimedio dell'art. 670 
Corte costituzionale che, con la sentenza n. 210 del 2013, ha demandato al giudice comune il compito «di determinare l'esatto campo di applicazione in sede esecutiva» delle «sopravvenienze relative alla punibilità e al trattamento punitivo del condannato», concludendo che, «in base all'art. 30, quarto comma, della legge n. 87 del 1953, il giudicato penale non impedisce al giudice di intervenire sul titolo esecutivo per modificare la pena, quando la misura di questa è prevista da una norma di cui è stata riconosciuta l'illegittimità convenzionale, e quando tale riconoscimento sorregge un giudizio altamente probabile di illegittimità costituzionale della norma per violazione dell'art. 117, primo comma, Cost.» ${ }^{66}$.

Questa subordinazione del ricorso all'incidente di esecuzione alla precedente declaratoria d'illegittimità costituzionale della norma sanzionatoria, è stata tuttavia posta in discussione dal secondo intervento delle Sezioni Unite della Suprema Corte nel "caso Ercolano", dove si è sostenuta l'attivabilità del «meccanismo di aggressione del giudicato, nella parte relativa alla specie e alla misura della pena inflitta dal giudice della cognizione», alle condizioni che sembra utile riprodurre qui testualmente: «a) la questione controversa deve essere identica a quella decisa dalla Corte EDU; b) la decisione sovranazionale, alla quale adeguarsi, deve avere rilevato un vizio strutturale della normativa interna sostanziale, che definisce le pene per determinati reati, in quanto non coerente col principio di retroattività in mitius; c) la possibilità d'interpretare la normativa interna in senso convenzionalmente orientato ovvero, se ciò non è possibile, la declaratoria d'incostituzionalità della medesima normativa (com'è accaduto nel caso di specie); d) l'accoglimento della questione sollevata deve essere l'effetto di una operazione sostanzialmente ricognitiva e non deve richiedere la riapertura del processo ${ }^{67}$. Peraltro, il

c.p.p., riguardante «l'ipotesi in cui il titolo manca o non è divenuto esecutivo, laddove nel caso in esame la sentenza di patteggiamento è sicuramente passata in giudicato e tale è divenuta prima che sopravvenisse l'illegalità della pena per effetto della dichiarazione di incostituzionalità, per cui non può neppure parlarsi di un giudicato "viziato". Non avrebbe alcun senso il ricorso all'art. 670 cod. proc. pen. con conseguente ritorno degli atti al giudice della cognizione: in questo caso, infatti, la sentenza è validamente passata in giudicato». Corte cost., 18 luglio 2013, n. 210, par. 7.3 motivazione.

67 Cass., Sez. Un., 7 maggio 2014, n. 18821, cit., par. 9.2 motivazione (corsivo aggiunto). 
riferimento alla possibilità d'interpretazione convenzionalmente orientata della legge nazionale, come strada da percorrere con priorità rispetto all'incidente davanti alla Corte costituzionale, sembrerebbe preludere a una riapplicazione della disciplina penale sostanziale da parte del giudice dell'esecuzione, che, come si è ricordato, è estranea ai compiti attribuiti a quest'organo, nei tassativi casi di rideterminazione della pena in deroga alla condanna divenuta irrevocabile ${ }^{68}$. Il superamento di questo principio dovrebbe pertanto passare da un intervento della Corte costituzionale (che estenda i casi di attivazione del giudice dell'esecuzione).

Inoltre, secondo il diritto vivente, la soluzione dell'incidente di esecuzione si attaglia a casi, come quello "Ercolano", in cui «non è necessaria una "riapertura del processo" di cognizione ma occorre più semplicemente incidere sul titolo esecutivo, in modo da sostituire la pena irrogata con quella conforme alla CEDU e già precisamente determinata nella misura dalla legge» ${ }^{69}$. Sostiene la Consulta che, "per una simile attività processuale», non è adeguato il procedimento di revisione previsto dall'art. 630 c.p.p., quale risulta a seguito della dichiarazione d'illegittimità costituzionale da parte della sentenza n. 113/2011 ${ }^{70}$, «ma è sufficiente», prosegue la Corte, «un intervento del giudice dell'esecuzione», «specie se si considera l'ampiezza dei poteri ormai riconosciuti dall'ordinamento processuale a tale giudice, che non si limita a conoscere delle questioni sulla validità e sull'efficacia del titolo esecutivo ma è anche abilitato, in vari casi, ad incidere su di esso (artt. 669, 670, comma 3, 671, 672 e 673 cod. proc. pen.)» $\gg^{71}$.

Sul riconoscimento normativo di tali ampi poteri ha poi fatto perno la giurisprudenza di legittimità per allargare gli spazi d'incidenza del rito esecutivo sul giudicato, al di là dei casi in cui la pena inflitta

68 In tal senso, v. LAVARINI, Barbara, I rimedi post iudicatum alla violazione dei canoni europei, cit., p. 112.

69 Corte cost., 18 luglio 2013, n. 210, par. 8 motivazione (corsivo aggiunto); nonché Cass., Sez. Un., 7 maggio 2014, n. 18821, cit., par. 8 motivazione: «il meccanismo procedurale da utilizzare non può che essere individuato nell'incidente di esecuzione».

70 Cass., Sez. Un., 7 maggio 2014, n. 18821, cit., par. 8 motivazione.

71 Corte cost., 18 luglio 2013, n. 210, par. 8 motivazione; Cass., Sez. Un., 7 maggio 2014, n. 18821, cit., par. 9 motivazione. 
debba esser rideterminata a schema fisso (come avvenuto nella "vicenda Ercolano" con la sostituzione della pena dell'ergastolo con quella della reclusione di trent'anni) o con operazione meramente aritmetica (come nell'evenienza in cui il giudice dell'esecuzione sia chiamato a detrarre dalla pena inflitta, «la quantità "illegale" di pena "aggiunta" per effetto di una circostanza aggravante dichiarata costituzionalmente illegittima» $)^{72}$. Secondo la "pronuncia Gatto" delle Sezioni Unite della Corte di cassazione, il giudice dell'esecuzione dovrebbe in generale rideterminare la pena «applicata per effetto di norme che mai avrebbero dovuto vivere nell'ordinamento», in quanto dichiarate incostituzionali ${ }^{73}$; e, quindi, anche quando oggetto della declaratoria di incostituzionalità non sia una circostanza aggravante, ma il divieto normativo di prevalenza delle circostanze attenuanti sull'aggravante prevista dall'art. 99 comma $4^{\circ}$ c.p. (come stabilito dall'art. 69 comma $4^{\circ}$ c.p., a seguito della $1 . n$. $251 / 2005)^{74}$. In base alla lettura della Suprema Corte, anche in un caso simile la pena sarebbe eseguita illegittimamente, innanzitutto sotto il profilo oggettivo, «in quanto derivante dall'applicazione di una norma di diritto penale sostanziale dichiarata incostituzionale dopo la sentenza irrevocabile» ${ }^{75}$. Inoltre, sotto il profilo soggettivo, la pena risulterebbe illegittima, «giacchè, almeno per una sua parte, non potrà essere positivamente finalizzata alla rieducazione del condannato e costituirà, anzi, un ostacolo al perseguimento di tale scopo perché sarà "inevitabilmente" avvertita come ingiusta da chi la sta subendo, per essere stata non già determinata dal giudice nell'esercizio dei suoi ordinari e legittimi poteri, ma imposta da un legislatore che ha violato la Costituzione» ${ }^{76}$.

Questo ragionamento sembra peraltro provare troppo, perché il condannato avvertirà "inevitabilmente" come "ingiusta", a scapito

72 È il problema che si è posto a seguito della declaratoria d'illegittimità costituzionale dell'art. 61 comma $1^{\circ}$, n. 11-bis), c.p. (sent. cost. n. 249/2010), ed è stato affrontato, ad es., da Cass., Sez. I, 13 gennaio 2012, n. 977, cit.

73 Cass., Sez. Un., 14 ottobre 2014, n. 42858, cit., par. 10.2 motivazione.

74 Cass., Sez. Un., 14 ottobre 2014, n. 42858, cit., par. 11 motivazione. La disposizione è stata dichiarata costituzionalmente illegittima da Corte cost., 15 novembre 2012, n. 251.

75 Cass., Sez. Un., 14 ottobre 2014, n. 42858, cit., par. 12 motivazione.

76 Cass., Sez. Un., 14 ottobre 2014, n. 42858 , cit., par. 12 motivazione. 
del principio rieducativo di cui all'art. 27 comma $3^{\circ}$ Cost., anche (e a maggior ragione) la pena eseguita in base a un giudizio di colpevolezza ad esempio scaturito dalla utilizzazione di una prova prevista da una norma processuale poi dichiarata costituzionalmente illegittima. Eppure, in una situazione di questo tipo, non vi sarebbe spazio per attivare la revisione, né l'incidente di esecuzione, senza ulteriore rimedio a tutela della proclamazione d'innocenza post iudicatum ${ }^{77}$.

Ancora, è stata portata all'attenzione delle Sezioni Unite, nel “caso Marcon", la questione se debba essere rideterminata in sede esecutiva la pena applicata su richiesta delle parti per i delitti previsti dall'art. 73 d.P.R. n. 309 del 1990, in relazione alle "droghe leggere", con pronuncia divenuta irrevocabile prima della sentenza della Corte costituzionale n. 32 del 2014, e, in caso di risposta affermativa, secondo quali regole debba essere rideterminata, cioè «secondo un criterio aritmetico-proporzionale di adeguamento alla mutata e più favorevole cornice edittale, o se, invece, il giudice dell'esecuzione possa rivalutare la congruità e la correttezza della sanzione irrogata dal giudice della cognizione rispetto alla disciplina oggetto di reviviscenza avvalendosi dei criteri discrezionali di cui agli artt. 132 e 133 cod. pen. $»^{78}$. Vale a dire se, in caso di risposta affermativa al primo quesito, possa essere esercitato da parte del giudice dell'esecuzione il potere discrezionale, tipicamente attribuito al giudice della cognizione nell'applicazione della pena, entro i limiti e secondo i criteri dettati nei due articoli richiamati (ben al di là, quindi, della messa a punto delle Sezioni Unite risalente al "caso Ercolano"). Ebbene la Consulta, dichiarata l'illegittimità costituzionale, in riferimento all'art. 77 comma $2^{\circ}$ Cost., delle modifiche all'art. 73 d.P.R. n. 309/1990, da parte del d.l. n. 272/2005, conv. con modif. dalla 1. n. 49/2006, ha ritenuto «che la disciplina dei reati sugli stupefacenti contenuta nel d.P.R. [cit.], nella versione precedente alla novella del 2006, torni ad applicarsi, non essendosi validamente verificato l'effetto abrogativo ${ }^{79}$, ed ha poi demandato al giudice comune

77 Oppure si pensi alle «sentenze irrevocabili di condanna che siano il frutto di una valutazione grossolanamente errata delle risultanze processuali (non aggredibili, in quanto tali, con il rimedio straordinario della revisione)»: CAPRIOLI, Francesco, Il giudice e la legge processuale: il paradigma rovesciato, cit., p. 977.

78 Cass., Sez. Un., 15 settembre 2015, n. 37107, in C.E.D. Cass., rv. 264859.

79 Corte cost., 25 febbraio 2014, n. 32, par. 5 motivazione. 
il compito di individuare la norma penale più favorevole al reo, tenuto conto del trattamento sanzionatorio più mite da parte del testo originario del d.P.R. n. 309/1990, rispetto a quello caducato, per gli illeciti concernenti le "droghe leggere" (puniti con la pena della reclusione da due a sei anni e della multa, anziché con la pena della reclusione da sei a venti anni e della multa), e del trattamento sanzionatorio più severo per i reati concernenti le "droghe pesanti" (puniti con la pena della reclusione da otto a venti anni, anziché con quella da sei a venti anni) ${ }^{80}$.

Dal canto suo, la "pronuncia Marcon" ha affermato in primo luogo che, nel caso dell'illegalità della pena sopravvenuta alla sentenza di patteggiamento irrevocabile ${ }^{81}$, a causa della pronuncia della Corte costituzionale, deve essere rimesso in discussione, non «il titolo esecutivo nella sua interezza, ma solo una parte di esso, cioè la quantificazione della pena» ${ }^{82}$ (di qui l'inapplicabilità dell'art. 673 c.p.p.); con la conseguenza che «la rideterminazione del trattamento sanzionatorio assume una valenza sostitutiva di un titolo esecutivo [...] solo in parte non più eseguibile, che andrà pertanto integrato, in punto di entità della pena, dalla decisione emessa in sede esecutiva» ${ }^{83}$. In secondo luogo, la decisione in discorso ha elaborato una complessa teoria relativa al meccanismo da assegnare al giudice dell'esecuzione, per rideterminare la pena alla luce della disciplina sanzionatoria come risultante dopo la dichiarazione d'illegittimità costituzionale ${ }^{84}$.

A tal fine non sarebbe impiegabile il «criterio oggettivo di tipo matematico-proporzionale», in forza del quale dovrebbe applicarsi «una pena che, in proporzione, corrisponda all'entità della pena applicata in sentenza,

80 Corte cost., 25 febbraio 2014, n. 32, par. 6 motivazione.

81 Per il diverso caso dell'illegalità sopravvenuta alla sentenza di condanna non irrevocabile, v. la sentenza "gemella" Cass., Sez. Un., 28 luglio 2015, n. 33040, in C.E.D. Cass., rv. 264207.

82 Cass., Sez. Un., 15 settembre 2015, n. 37107, cit., par. 5.2 motivazione.

83 Cass., Sez. Un., 15 settembre 2015, n. 37107, cit., par. 5.2 motivazione.

${ }^{84}$ Qualora, invece, la dichiarazione d'incostituzionalità preceda la formazione del giudicato, Cass., Sez. Un., 28 luglio 2015, n. 33040, cit., par. 7 motivazione, ha sostenuto che «l'accordo è nullo per sopravvenuta illegalità di una parte del suo oggetto, con l'ulteriore conseguenza che la sentenza che ha recepito l'accordo deve essere annullata senza rinvio» (senza possibilità di rideterminazione d'ufficio). 
aggiungendo al nuovo minimo di pena la stessa percentuale di aumento applicata in sede di cognizione». Questo criterio, che secondo l'indirizzo giurisprudenziale dove è stato recepito salvaguarderebbe «la volontà negoziale delle parti irrevocabilmente espressa e la susseguente valutazione di congruità della pena concordata espressa dal giudice della cognizione» (e quindi preserverebbe maggiormente il giudicato $)^{85}$, secondo la pronuncia "Marcon" non sarebbe coerente ai «principi affermati dalle sentenze "Ercolano" e "Gatto" che, invece, riconoscono al giudice della esecuzione ampi poteri e margini di apprezzamento ${ }^{86}$. Né sarebbe applicabile il meccanismo basato sulla «tesi della libera determinazione della pena da parte del giudice dell'esecuzione», «in termini ormai sottratti alla dinamica negoziale», con il rischio, secondo la stessa "sentenza Marcon", «di applicare al condannato una pena ex art. $444 \mathrm{cod}$. proc. pen. di fatto non concordata ${ }^{87}$.

Nella lettura della Suprema Corte, sarebbe invece utilizzabile lo strumento processuale previsto dall'art. 188 n. att. c.p.p. in tema di applicazione della disciplina del concorso formale e del reato continuato da

85 V. i richiami giurisprudenziali effettuati dalle Sezioni Unite.

86 Cass., Sez. Un., 15 settembre 2015, n. 37107, cit., par. 7.1 motivazione, sottolineando che «l'originaria valutazione di congruità della pena irrogata, sia nella commisurazione della sanzione effettuata in sede di giudizio di colpevolezza, sia nel giudizio di congruità rimesso al giudice in caso di patteggiamento, è intimamente connessa alla diversa forbice edittale, all'epoca unica per tutti i tipi di sostanze stupefacenti, sicchè un criterio automatico di riduzione proporzionale finisce per non tenere conto della concreta gravità dei fatti e della personalità del reo in rapporto alla nuova cornice edittale». Ciò è coerente all'affermazione di Cass., Sez. Un., 28 luglio 2015, n. 33040, cit., par. 5 motivazione, secondo cui «il venir meno per contrarietà alla Costituzione con efficacia ex tunc - della cornice edittale che ha guidato il giudicante nella delicata attività di "misurazione della responsabilità" finisce con il travolgere la stessa pena in concreto inflitta, vale a dire il "risultato finale" di detta misurazione, perché, non essendo più attuale il giudizio astratto di disvalore del fatto (essendosi modificata la forbice sanzionatoria edittale), la misurazione compiuta non traduce più - per effetto del mutamento dei parametri di riferimento - né coerentemente né correttamente il giudizio di responsabilità». Prosegue la Suprema Corte osservando che, «in altri termini, la valutazione di responsabilità del reato non risulta più misurata "legalmente", perché la risposta punitiva è stata elaborata sulla base di un compasso sanzionatorio incostituzionale, così da risultare alterato lo stesso giudizio di gravità del reato ai sensi e per gli effetti di cui agli artt. 132 e 133 cod. pen.».

Cass., Sez. Un., 15 settembre 2015, n. 37107, cit., par. 7.2 motivazione. 
parte del giudice dell'esecuzione, nel caso di più sentenze di applicazione della pena su richiesta delle parti: la disposizione, ritenuta speciale rispetto all'art. 671 c.p.p., avrebbe l'identica ratio di «assicurare l'applicazione della pena "giusta" per l'imputato fino alla fase esecutiva» ${ }^{88}$. È la stessa Corte ad esplicitare, al riguardo, la necessità d'interpretare analogicamente l'art. 188 n. att. c.p.p. ${ }^{89}$, il che appare coerente alle richiamate affermazioni della giurisprudenza "Ercolano", ma si pone in rapporto critico con il principio di tassatività delle ipotesi derogatorie del giudicato ${ }^{90}$.

È poi importante sottolineare che, secondo la decisione in discorso, il giudice dell'esecuzione conserverebbe il potere di valutare la congruità della pena, ma anche di rideterminarla autonomamente nel caso essa sia ritenuta incongrua, in quanto, se così non fosse, «l'effetto sarebbe quello di confermare una pena illegale, laddove al giudice dell'esecuzione si chiede, con l'instaurazione dell'incidente, di rimuovere l'illegalità della pena oggetto dell'accordo originario»» ${ }^{91}$. Questo potere di «riqualificazione sanzionatoria», per un verso, è «sempre subordinato al previo esperimento di un tentativo di patteggiamento, fallito il quale, a causa di una pena "incongrua" rispetto al fatto così come ritenuto in sede di cognizione, spetta al giudice operare la modifica della sanzione»; per altro verso, tale potere dovrà essere esercitato dal giudice dell'esecuzione utilizzando i criteri di cui agli artt. 132 e 133 c.p..$^{92}$, dunque secondo la discrezionalità tipica del giudizio di cognizione.

Analogamente, il giudice dell'esecuzione dovrà valutare l'applicabilità della sospensione condizionale della pena, che le parti potrebbero includere nell'accordo, ma nel caso in cui non condivida l'applicazione del beneficio, «non potendo respingere l'accordo, come invece avviene in sede di cognizione, dovrà comunque recepirlo, escludendo la sospensione condizionale, sempre che ritenga congrua la pena. Allo stesso

88 Cass., Sez. Un., 15 settembre 2015, n. 37107, cit., par. 8 motivazione. Cass., Sez. Un., 15 settembre 2015, n. 37107, cit., par. 8 motivazione. Ciò consentirebbe «di intervenire sulla pena illegale della sentenza di patteggiamento irrevocabile», e assicurerebbe «alle parti la possibilità di rinnovare l'accordo, rispettando l'essenza stessa dell'istituto dell'applicazione della pena su richiesta»: Cass., Sez. Un., 15 settembre 2015, n. 37107, cit., par. 8 motivazione. Cass., Sez. Un., 15 settembre 2015, n. 37107, cit., par. 8.2 motivazione. Cass., Sez. Un., 15 settembre 2015, n. 37107, cit., par. 8.3 motivazione. 
modo, deve riconoscersi che anche nelle residuali ipotesi di autonoma rideterminazione della pena il giudice possa disporre la sospensione condizionale della pena ${ }^{93}$. Come si vede, il superamento del giudicato porterebbe persino a un allargamento dei margini di manovra del giudice dell'esecuzione, rispetto al giudice della cognizione.

\section{L'INTANGIBILITÀ DEL GIUDICATO NELLA GIURISPRUDENZA DELLA CORTE DI CASSAZIONE}

La ritenuta attivabilità in sede esecutiva dei poteri discrezionali con cui il giudice determina la pena ex artt. 132 e 133 c.p., secondo la ricostruzione giurisprudenziale anzidetta, stride con la lettura fornita dalle stesse Sezioni Unite della Corte di cassazione, con la sentenza "Basile", circa i «limiti» e l'«ambito dell'intervento sul giudicato da parte del giudice dell'esecuzione» quando sia in gioco il problema di dover emendare in executivis una pena accessoria illegale ${ }^{94}$, «anche nel caso in cui debba essere eseguita una pena $a b$ origine illegale ${ }^{95}$ (cioè a prescindere dalla sopravvenienza di una declaratoria d'illegittimità costituzionale della disciplina penale relativa al trattamento sanzionatorio). Ha sostenuto la Corte, al riguardo, che «l'intervento del giudice dell'esecuzione è ammesso sempre che non implichi valutazioni discrezionali in ordine alla specie ed alla durata della pena accessoria ${ }^{96}$.

93 Cass., Sez. Un., 15 settembre 2015, n. 37107, cit., par. 9 motivazione, argomentando dal potere espressamente attribuito al giudice dell'esecuzione dall'art. 671 comma 3 c.p.p.

94 Cass., Sez. Un., 12 febbraio 2015, n. 6240, in C.E.D. Cass., rv. 262328, par. 6 motivazione.

95 Cass., Sez. Un., 12 febbraio 2015, n. 6240, cit., par. 3.3 motivazione. La Suprema Corte, in questa pronuncia, ha affrontato la questione «se l'erronea o omessa applicazione da parte del giudice della cognizione di una pena accessoria predeterminata per legge nella specie e nella durata o l'applicazione da parte del medesimo giudice, previa delimitazione del principio di legalità della pena in rapporto al giudicato e alla sua applicazione in sede esecutiva, di una pena accessoria extra o contra legem, possano essere rilevate, anche dopo il passaggio in giudicato della sentenza, dal giudice dell'esecuzione».

96 Cass., Sez. Un., 12 febbraio 2015, n. 6240, cit., par. 6.2 motivazione (corsivo aggiunto). 
Questa tesi ${ }^{97}$ è stata argomentata sia in base al disposto dell'art. 183 n. att. c.p.p., che attribuisce al pubblico ministero il dovere di chiedere l'applicazione della pena accessoria "al giudice dell'esecuzione se non si è provveduto con la sentenza di condanna", quando si tratta di "una pena accessoria predeterminata dalla legge nella specie e nella durata"; sia in base a considerazioni sistematiche attinenti ai limiti che incontrano i poteri dell'organo giurisdizionale nella fase esecutiva, di modo da escludere «interventi che comportino valutazioni di carattere discrezionale ${ }^{98}$ : non è in particolare consentita, secondo la ricostruzione in discorso, la manipolazione del giudicato attraverso «il ricorso ai criteri di cui all'art. 133 cod. pen., per la determinazione della durata della pena accessoria ${ }^{99}$. La Suprema Corte ha pertanto enunciato il principio di diritto per cui «l'applicazione di una pena accessoria extra o contra legem da parte del giudice della cognizione può essere rilevata, anche dopo il passaggio in giudicato della sentenza, dal giudice dell'esecuzione, purchè essa sia determinata per legge (o determinabile, senza alcuna discrezionalità) nella specie e nella durata, e non derivi da un errore valutativo del giudice della cognizione».

Non può stupire il riferimento a questo limite negativo dell'intervento sul giudicato. Infatti, la costante giurisprudenza di legittimità esclude che il giudicato sia emendabile quando l'applicazione di una pena illegale scaturisca da un accertamento sul punto, sia pure erroneo, del giudice della cognizione ${ }^{100}$. La stessa "pronuncia Gatto" delle Sezioni Unite ha posto l'accento su questa forma di manifestazione dell'“intangibilità" della pronuncia irrevocabile, nonostante l'intervenuta declaratoria d'illegittimità costituzionale di una norma penale idonea a mitigare il trattamento sanzionatorio. Circoscrivendo gli effetti

97 Per una critica, v. LAVARINI, Barbara, I rimedi post iudicatum alla violazione dei canoni europei, cit., pp. 114-115.

Cass., Sez. Un., 12 febbraio 2015, n. 6240, cit., par. 6.2 motivazione.

99 Cass., Sez. Un., 12 febbraio 2015, n. 6240, cit., par. 6.3 motivazione.

${ }^{100}$ Cass., Sez. Un., 12 febbraio 2015, n. 6240, cit., par. 6.1 motivazione, secondo cui va appunto «esclusa l'emendabilità in executivis quando il giudice della cognizione si sia già pronunciato in proposito e sia pervenuto, anche se in modo erroneo, a conclusioni che abbiano comportato l'applicazione di una pena accessoria illegale. In tal caso, alla erroneità della valutazione non può che porsi rimedio con gli ordinari mezzi di impugnazione». 
della già richiamata sentenza costituzionale n. 251/2012, in ordine al divieto ex art. 69 comma $4^{\circ}$ c.p. di valutare prevalente la circostanza attenuante di cui all'art. 73 comma 5 d.P.R. n. 309/1990, sulla recidiva ex art. 99 comma $4^{\circ}$ c.p., la Suprema Corte ha affermato il potere del giudice dell'esecuzione di ritenere la suddetta prevalenza, «semprechè una simile valutazione non sia stata esclusa nel merito dal giudice della cognizione, secondo quanto risulta dal testo della sentenza irrevocabile» ${ }^{101}$. Si è così individuato, nella sentenza di cognizione, un vero e proprio "limite" di rideterminazione della pena da parte del giudice dell'esecuzione, le cui valutazioni «non potranno contraddire quelle del giudice della cognizione» (come nel caso in cui il giudizio di prevalenza sia stato escluso "per ragioni di merito, cioè indipendentemente dal divieto previsto dall'art. 69, quarto comma, cod. pen.») ${ }^{102}$.

È necessario infine richiamare un ulteriore passaggio dell'elaborazione giurisprudenziale sui limiti d'intervento sul giudicato penale. Esso non può cedere, e resta dunque intangibile, pur di fronte a violazioni del principio di legalità, quando la pena sia stata del tutto espiata (o si sia estinta). Questo presupposto di attivazione in executivis dei rimedi alle suddette violazioni è stato enucleato dalla Suprema Corte, in forza dell'esigenza di rendere il giudicato stesso «recessivo di fronte ad evidenti e pregnanti compromissioni in atto dei diritti fondamentali della persona» ${ }^{103}$, con «effetti», quindi, «ancora perduranti della violazione» ${ }^{104}$. Ove sia ancora attuale l'esecuzione della pena illegale, non vi sarebbe al contrario spazio per invocare «l'avvenuto esaurimento del rapporto, che secondo la giurisprudenza costituzionale e di legittimità costituisce il limite della retroattività della declaratoria di illegittimità costituzionale» ${ }^{105}$.

${ }^{101}$ Così il principio di diritto enunciato da Cass., Sez. Un., 14 ottobre 2014, n. 42858, cit.

102 Cass., Sez. Un., 14 ottobre 2014, n. 42858, cit., par. 12 motivazione. Cfr. anche Cass., Sez. Un., 15 settembre 2015, n. 37107, cit., par. 4 motivazione, secondo cui «il limite all'opera di rideterminazione della pena da parte del giudice dell'esecuzione, che può fare uso di poteri istruttori, è costituito da quanto già accertato dal giudice di cognizione per ragioni di merito, cioè da quanto accertato non facendo applicazione della norma dichiarata incostituzionale».

103 Cass., Sez. Un., 7 maggio 2014, n. 18821, cit., par. 7.1 motivazione.

${ }^{104}$ Cass., Sez. Un., 7 maggio 2014, n. 18821, cit., par. 7.1 motivazione.

105 Così, Cass., Sez. Un., 14 ottobre 2014, n. 42858, cit., par. 8.1 motivazione. 
È stata, ancora, la "sentenza Gatto" della Corte di cassazione a individuare il fondamento normativo della tesi, affermando che «l'aspetto decisivo, che segna [...] il limite non discutibile di impermeabilità e insensibilità del giudicato anche alla situazione di sopravvenuta declaratoria di illegittimità costituzionale della norma applicata, è costituito dalla non reversibilità degli effetti, giacchè l'art. 30 legge n. 87 del 1953 impone di rimuovere tutti gli effetti pregiudizievoli del giudicato non divenuti nel frattempo irreversibili, ossia quelli che non possono essere rimossi, perché già "consumati”, come nel caso di condannato che abbia già scontato la pena» ${ }^{106}$. In quest'ottica, ha peraltro osservato la dottrina processuale, vi sarebbe un mutamento di funzione dell'art. 30 comma 4, «che perde la fisionomia di deroga all'intangibilità della res iudicata per rivestire un ruolo meramente attuativo [della decisione della Corte costituzionale], nella misura in cui obbliga a rimuovere le conseguenze pregiudizievoli della norma incostituzionale. È da simile prospettiva che - ad avviso delle Sezioni unite - si ricava il limite estremo alla permeabilità del giudicato: ove la pena risulti espiata, gli effetti verificatisi diventano ormai irreversibili; solo allora il rapporto esecutivo non è più “attuale” ${ }^{107}$.

${ }^{106}$ Cass., Sez. Un., 14 ottobre 2014, n. 42858, cit., par. 8.1 motivazione, osservando ulteriormente che «l'esecuzione della pena, infatti, implica esistenza di un rapporto esecutivo che nasce dal giudicato e si esaurisce soltanto con la consumazione o l'estinzione della pena. Sino a quando l'esecuzione della pena è in atto, per definizione il rapporto esecutivo non può ritenersi esaurito e gli effetti della norma dichiarata costituzionalmente illegittima sono ancora perduranti e, dunque, possono e devono essere rimossi». In questa pronuncia la Suprema Corte ha pertanto enunciato il principio di diritto secondo cui «successivamente a una sentenza irrevocabile di condanna, la dichiarazione d'illegittimità costituzionale di una norma penale diversa dalla norma incriminatrice, idonea a mitigare il trattamento sanzionatorio, comporta la rideterminazione della pena, che non sia stata interamente espiata, da parte del giudice dell'esecuzione» (corsivo aggiunto). Nel senso che «la legalità della pena deve essere sempre costantemente assicurata, anche nella fase dell'esecuzione - almeno fino a quando non sia stata interamente eseguita», v. anche Cass., Sez. Un., 15 settembre 2015, n. 37107, cit., par. 6 motivazione (corsivo aggiunto).

107 VICOLI, Daniele, L'illegittimità costituzionale della norma penale sanzionatoria travolge il giudicato, cit., pp. 1013-1014. 


\section{LA RESISTENZA DEL GIUDICATO ALL'OVERRULING GIURISPRUDENZIALE FAVOREVOLE E IL RISPETTO DEL NULLUM CRIMEN}

Ritornando alla tesi che subordina la derogabilità del giudicato all'assenza di un'erronea valutazione del giudice nella fase cognitiva ${ }^{108}$, merita ricordare che un possibile temperamento del principio potrebbe esser ricavato da un'applicazione estesa dell'art. 673 c.p.p. ai fenomeni di abolitio criminis. Per come formulata, la disposizione processuale sembrerebbe coprire tanto il caso (già considerato dall'art. 2 comma $2^{\circ}$ c.p.) in cui l'abrogazione della norma incriminatrice sia sopravvenuta alla formazione del giudicato, quanto il caso in cui l'abrogazione preceda la condanna o, persino, il fatto commesso dall'imputato, «rappresentando, cioè, un rimedio per il palese errore giudiziario» ${ }^{109}$.

Da questo dato di legge ha tratto spunto una parte della dottrina per sostenere l'attivabilità del rimedio da parte del giudice dell'esecuzione, nel caso in cui il giudice della cognizione «non si fosse avveduto della già intervenuta abrogazione tacita della norma incriminatrice o anche se consapevolmente non avesse ritenuto di ravvisare nella successione di leggi il fenomeno dell'abrogazione tacita ${ }^{110}$. Vi sarebbe quindi spazio per sindacare in executivis l'errore di giudizio compiuto nella fase della cognizione, superando, in base alla formulazione dell'art. 673 c.p.p., le obiezioni avanzate da altra parte della dottrina contro l'ipotesi estensiva ${ }^{111}$. In tal modo, l'obiettivo di tutelare il principio del nullum crimen sine lege, anche in deroga al giudicato penale, verrebbe perseguito, non già tramite

108 V., supra, par. 5.

${ }^{109}$ MAZZA, Oliviero, Principio di legalità, diritto giurisprudenziale e giudice dell'esecuzione, cit., p. 205. V. anche VICOLI, Daniele, Il giudice dell'esecuzione, in CAPRIOLI, Francesco; VICOLI, Daniele, Procedura penale dell'esecuzione, cit., p. 281, che ritiene «consentito l'intervento "riparatore"», sia pure entro precisi limiti: «se il fatto era già descritto in termini tali da ricadere nell'area del penalmente lecito e la causa di proscioglimento è sfuggita al giudice, l'art. 673 c.p.p. offre il rimedio necessario a sanare l'illegalità della sanzione irrogata».

${ }^{110}$ MAZZA, Oliviero, op. loc. cit.

${ }^{111}$ V., ad es., VICOLI, Daniele, op. loc. cit., secondo cui «la tesi negativa s'impone ove l'ipotesi dell'abolitio sia stata valutata ma poi esclusa dal giudice della cognizione: si ammetterebbe, infatti, il potere di sindacare, nella fase esecutiva, questioni di merito già coperte dal formarsi della res iudicata». 
interpretazioni analogiche (precluse dalla tassatività dei casi d'intervento in executivis), ma applicando direttamente l'art. 673 c.p.p., che così come vieta di «continuare a punire o anche solo a ritenere colpevole un soggetto per un fatto previsto dalla legge come reato al tempo della commissione e successivamente non più considerato tale dall'ordinamento», «allo stesso modo, e verrebbe da dire, a maggior ragione, [vieta di] continuare a punire o anche solo a ritenere colpevole chi ha tenuto una condotta che non era prevista dalla legge come reato già al momento della sua realizzazione» ${ }^{112}$.

Un fenomeno di abolizione tacita della norma incriminatrice, che secondo la ricostruzione in discorso avrebbe dovuto condurre all'applicazione dell'art. 673 c.p.p., stava alla base della sentenza n. 230/2012 con cui la Corte costituzionale ha peraltro dichiarato l'infondatezza della questione di legittimità della disposizione anzidetta, sollevata, in riferimento agli artt. $3,13,25$ comma $2^{\circ}, 27$ comma $3^{\circ}$ e 117 comma $1^{\circ}$ Cost., nella parte in cui non include, tra le ipotesi di revoca della sentenza di condanna (nonché del decreto penale e della sentenza di applicazione della pena su richiesta delle parti), anche il "mutamento giurisprudenziale", determinato da una decisione delle Sezioni Unite della Corte di cassazione, in base al quale il fatto giudicato non è previsto dalla legge come reato $^{113}$. Si discuteva in particolare, nel giudizio a quo, della contravvenzione di omessa esibizione di documenti, prevista dall'art. 6 comma 3 d.lgs. n. 286/1998, come modificato dalla 1. n. 94/2009, disposizione che, in origine, contemplava il reato anche per la condotta degli stranieri illegalmente presenti nel territorio dello Stato (venivano puniti gli stranieri che, "senza giustificato motivo", non esibiscono, a richiesta degli ufficiali e degli agenti di polizia di sicurezza, il passaporto o altro documento di identificazione, "ovvero" il permesso o la carta di soggiorno). Dopo l'intervento del 2009, e la sostituzione della disgiuntiva "ovvero" con la

${ }^{112}$ MAZZA, Oliviero, op. cit., p. 209, il quale prosegue osservando che «non è rilevante il tempo dell'abolitio criminis: nessuno può essere punito o ritenuto colpevole per un fatto non previsto dalla legge come reato. Tale principio discende direttamente dall'art. 25, comma 2, Cost. e dall'art. 7, comma 1, CEDU, e trova attuazione nell'art. 2, comma 2, c.p., per l'abolitio criminis sopravvenuta alla commissione del reato, e nell'art. 673 c.p.p., tanto per l'abolitio criminis successiva alla formazione del giudicato quanto per quella precedente tale momento o addirittura precedente il tempus commissi delicti».

${ }^{113}$ Corte cost., 8 ottobre 2012, n. 230. 
congiunzione "e", si era verificato un contrasto giurisprudenziale sulla valenza delle modifiche alla disposizione penale (meramente formale oppure sostanziale), il che aveva portato all'intervento delle Sezioni Unite della Suprema Corte e alla recezione della tesi per cui «la novella legislativa del 2009 avrebbe [...] comportato l'abolizione, ai sensi dell'art. 2, secondo comma, del codice penale, della fattispecie criminosa preesistente, per la parte in cui si indirizzava agli stranieri in posizione irregolare (Cass., sez. un., 24 febbraio 2011-27 aprile 2011, n. 16453)»114.

La Corte costituzionale ha individuato in questa vicenda un fenomeno di "mutamento giurisprudenziale", di cui ha peraltro affermato l'«estraneità [...] all'area applicativa dell'istituto della "revoca della sentenza per abolizione del reato”, quale attualmente delineato dall'art. 673 cod. proc. pen.» ${ }^{115}$, restringendo quindi la sfera operativa dell'istituto processuale rispetto alla ricostruzione dottrinale cui si è sopra fatto cenno. Dopodichè la Consulta ha escluso la fondatezza della questione di legittimità dedotta, ma non perché fosse erroneo il presupposto dell'ordinanza di rimessione ${ }^{116}$, che si prefiggeva di colmare l'assunto vuoto di tutela nell'art. 673 c.p.p., individuando una nuova deroga all'intangibilità del giudicato (la revoca della sentenza per abolizione del reato causata da overruling giurisprudenziale). La sentenza n. 230/2012 ha invece contestato il sillogismo effettuato dal giudice a quo, sulla scorta di due premesse peraltro fondate, vale a dire: l'affermazione per cui l'art. 7 CEDU, quale "norma convenzionale interposta" con cui l'art. 673 c.p.p. confliggerebbe, «sancisce implicitamente», secondo la recente giurisprudenza della Corte EDU, «anche il principio di retroattività della legge penale più mite»; l'ulteriore affermazione per cui «la nozione di "diritto" ("law"), utilizzata nella norma della Convezione, deve considerarsi comprensiva tanto del diritto di produzione legislativa che del diritto di formazione giurisprudenziale», in base alla consolidata giurisprudenza della stessa Corte di Strasburgo ${ }^{117}$.

\footnotetext{
${ }^{114}$ V. Corte cost., 8 ottobre 2012, n. 230, par. 2 motivazione

115 V. Corte cost., 8 ottobre 2012, n. 230, par. 5 motivazione

${ }^{116}$ V., invece, in questo senso, MAZZA, Oliviero, Principio di legalità, cit., pp. 203 ss.

117 Corte cost., 8 ottobre 2012, n. 230, par. 7 motivazione
} 
La Corte costituzionale ha appunto escluso «il corollario che il giudice a quo vorrebbe far discendere dalla combinazione tra i due asserti dinanzi ricordati, e, cioè, che in base all'art. 7, paragrafo 1, della CEDU, un mutamento di giurisprudenza in senso favorevole al reo imponga la rimozione delle sentenze di condanna passate in giudicato contrastanti col nuovo indirizzo» ${ }^{118}$. In questa direzione la Consulta ha argomentato non solo che «la Corte di Strasburgo non ha mai sinora riferito, in modo specifico, il principio di retroattività della lex mitior ai mutamenti di giurisprudenza», ma anche in base al «diverso fondamento» del principio di retroattività della lex mitior, rispetto a quello dell'irretroattività della norma sfavorevole, intesa quale «strumento di garanzia del cittadino contro persecuzioni arbitrarie, espressivo dell'esigenza di “calcolabilità" delle conseguenze giuridico-penale della propria condotta, quale condizione necessaria per la libera determinazione individuale: esigenza con la quale contrasta un successivo mutamento peggiorativo "a sorpresa" del trattamento penale della fattispecie» ${ }^{119}$. Secondo la Corte costituzionale, tale esigenza non presenta collegamenti con il principio di retroattività della legge più favorevole (a sua volta fondato sul principio di eguaglianza e con operatività limitata alle modifiche normative che intervengano prima della pronuncia definitiva) ${ }^{120}$.

Tuttavia, una volta che si riconosca l'applicabilità dell'art. 673 c.p.p. anche ai casi di abrogazione tacita della norma incriminatrice, dove è per definizione necessaria, a differenza dei casi di abrogazione espressa della disposizione penale, la «mediazione intellettuale dell'interprete», e quindi l'intervento della giurisprudenza ${ }^{121}$, tanto più quando l'effetto abrogativo «non sia agevolmente desumibile dal teso della legge posteriore ${ }^{122}$, non occorre più ipotizzare che l'eventuale overruling giu-

\footnotetext{
${ }^{118}$ Corte cost., 8 ottobre 2012, n. 230, par. 7 motivazione

119 Corte cost., 8 ottobre 2012, n. 230, par. 7 motivazione.

${ }^{120}$ Corte cost., 8 ottobre 2012, n. 230, par. 7 motivazione, sottolineando ulteriormente che «la limitazione ora indicata non potrebbe evidentemente non valere $[\ldots]$ anche in rapporto ai mutamenti di giurisprudenza».

${ }^{121}$ V. MAZZA, Oliviero, Principio di legalità, cit., pp. 206-207.

${ }^{122}$ Cass., Sez. Un., 23 giugno 2016, n. 26259, in C.E.D. Cass., rv. 266872, dove si osserva che, in tal caso, «è fisiologico che si sviluppi un'attività ermeneutica diretta a verificare la compatibilità tra le nuove disposizioni,
} 
risprudenziale possegga una rilevanza autonoma, rispetto al mutamento legislativo. La sentenza irrevocabile di condanna basata sulla norma penale previgente dovrà essere revocata a prescindere dal riconoscimento tardivo dell'abolitio in sede giurisprudenziale (e quindi a prescindere dal mutamento di giurisprudenza). Non si tratta, pertanto, di riconoscere un ulteriore profilo di "cedevolezza" del giudicato, di fronte ai cambiamenti del "diritto giurisprudenziale"123: è la legge processuale a far prevalere sull'intangibilità del giudicato la tutela del principio di legalità penale.

o alcune di esse, e quelle precedenti. Può quindi accadere che si determini un contrasto giurisprudenziale, con la conseguenza che l'effetto abrogativo su singole norme incriminatrici sia "riconosciuto" dal giudice per via interpretativa, anche a distanza di tempo dall'entrata in vigore della nuova disciplina».

${ }^{123} \mathrm{Si}$ è affermato condivisibilmente, nella citata sentenza costituzionale n. $230 / 2012$, che «non può ritenersi manifestamente irrazionale che il legislatore, per un verso, valorizzi, anche in ossequio ad esigenze di ordine costituzionale, la funzione nomofilattica della Corte di cassazione, e delle Sezioni unite in particolare [...] e, dall'altro, ometta di prevedere la revoca delle condanne definitive pronunciate in relazione a fatti che, alla stregua di una sopravvenuta diversa decisione dell'organo della nomofilachia, non sono previsti dalla legge come reato, col risultato di consentire trattamenti radicalmente differenziati di autori di fatti analoghi» (par. 9 motivazione). Osserva la Consulta, a sostegno di quanto detto, che «l'orientamento espresso dalla decisione delle Sezioni Unite "aspira" indubbiamente ad acquisire stabilità e generale seguito: ma [...] si tratta di connotati solo "tendenziali", in quanto basati su una efficacia non cogente, ma di tipo essenzialmente "persuasivo". Con la conseguenza che, a differenza della legge abrogativa e della declaratoria di illegittimità costituzionale, la nuova decisione dell'organo della nomofilachia resta potenzialmente suscettibile di essere disattesa in qualunque tempo e da qualunque giudice della Repubblica, sia pure con l'onere di adeguata motivazione; mentre le stesse Sezioni unite possono trovarsi a dover rivedere le loro posizioni, anche su impulso delle sezioni singole, come in più occasioni è in fatto accaduto. In questa logica si giustifica, dunque, il mancato riconoscimento all'overruling giurisprudenziale favorevole della capacità di travolgere il principio di intangibilità della res iudicata, espressivo dell'esigenza di certezza dei rapporti giuridici esauriti». In senso adesivo, sul punto, Cass., Sez. Un., 23 giugno 2016, n. 26259, cit., par. 6.1 motivazione, ma con importanti aperture alla ricostruzione dottrinale già richiamata nel testo, e, quindi, riconoscendo, a differenza della sentenza costituzionale n. 230/2012, che l'intervento delle Sezioni Unite con la sentenza n. 16453 del 2011, ha avuto «una funzione meramente ricognitiva dell'intervenuto effetto abrogativo parziale» della disciplina penale previgente alle modifiche del 2009. E di seguito: «La parziale abrogazione, 
È particolarmente significativo il riconoscimento della validità di quest'impostazione, cui è pervenuta una recente pronuncia delle Sezioni Unite della Corte di cassazione (vicenda "Mraidi"), con riferimento al caso della condanna per un fatto che non fosse più previsto dalla legge come reato già al momento della sua commissione ${ }^{124}$, dopo aver ricordato che «nell'art. 673 cod. proc. pen. non sono previste limitazioni ai poteri di accertamento e di valutazione del giudice dell'esecuzione, a differenza di quanto previsto dall'art. 671 (applicazione del concorso formale e del reato continuato in sede esecutiva) e 675 cod. proc. pen. (dichiarazione della falsità di documenti in sede esecutiva) che prevedono una riserva in favore del giudice della cognizione» ${ }^{125}$. Il compito di verificare, ai sensi dell'art. 673 c.p.p., l'eventuale "abrogazione della norma incriminatrice" attribuisce al giudice dell'esecuzione, secondo la Corte di cassazione, un ruolo di «garante della legalità penale», in funzione del rispetto del principio nullum crimen, nulla poena sine lege, con la conseguenza che, prosegue la "sentenza Mraidi", «non vi è ragione di circoscrivere le ipotesi di revoca per abolitio disciplinate dall'art. 673 cod. proc. pen. ai casi previsti dall'art. 2, secondo comma, cod. pen. e non anche a quelli del primo comma ("Nessuno può essere punito per un fatto che, secondo la legge del tempo in cui fu commesso, non costituiva

benchè riconosciuta e affermata dalle Sezioni Unite nell'esercizio della funzione nomofilattica che è propria della Corte di cassazione, rimane infatti un atto di volontà novativa del legislatore che, come rilevato in dottrina, nel nostro assetto costituzionale è l' "unico metro di riconoscimento degli effetti abrogativi di una norma”. Diverso sarebbe stato il caso in cui, in assenza di interventi normativi, si fossero susseguite interpretazioni giurisprudenziali diverse dello stesso testo legislativo, poiché [...] il precetto fondamentale della soggezione del giudice soltanto alla legge (art. 101 Cost.) impedisce di attribuire all'interpretazione della giurisprudenza il valore di fonte del diritto, sicchè essa, nella sua dimensione dichiarativa, non può rappresentare la lex temporis acti, ossia il parametro normativo immanente per la verifica di validità dell'atto compiuto in correlazione temporale con l'affermarsi dell'esegesi del giudice».

${ }^{124}$ Per il caso, solo in parte diverso, della condanna per un fatto che non fosse più previsto dalla legge come reato al momento del passaggio in giudicato della sentenza, v. invece, ad es., Cass., Sez. I, 14 gennaio 2015, n. 1611, in C.E.D. Cass., rv. 261984.

125 Cass., Sez. Un., 23 giugno 2016, n. 26259, cit., par. 6.2 motivazione. 
reato"), che traggono valore cogente dall'art. 25, secondo comma, Cost. ("Nessuno può essere punito se non in forza di una legge che sia entrata in vigore prima del fatto commesso")» ${ }^{126}$.

Di qui l'enunciazione del principio di diritto per cui il giudice dell'esecuzione «può revocare», ai sensi del citato art. 673 c.p.p., «una sentenza di condanna pronunciata dopo l'entrata in vigore della legge che ha abrogato la norma incriminatrice, allorchè l'evenienza di abolitio criminis non sia stata rilevata dal giudice della cognizione». L'inciso è da intendersi come una sorta di restrizione della portata pratica del principio di diritto, che la "sentenza Mraidi" ha operato richiamando la tesi della "pronuncia Basile" delle stesse Sezioni Unite ${ }^{127}$, in ordine al limite negativo per derogare al giudicato costituito dall'assenza di un errore valutativo del giudice della cognizione (irrimediabile in executivis). Secondo la Corte, «la mancanza nella motivazione della sentenza di condanna di qualunque valutazione circa l'eventualità di una parziale abolitio criminis della norma incriminatrice induce a ritenere, in assenza di ulteriori elementi desumibili dal contesto della motivazione, che il giudice della cognizione [...] non sia incorso in un errore "valutativo"», potendo semmai ravvisarsi «nel silenzio del giudice della cognizione un mero errore percettivo» ${ }^{128}$.

Contro l'ipotesi di applicare l'art. 673 c.p.p., per rimediare alla violazione dell'art. 7 CEDU accertata in una pronuncia della Corte di Strasburgo, si è tuttavia espressa la Corte di cassazione recentemente, in ordine al diverso problema del giudicato di condanna per un fatto che non fosse ancora previsto dalla legge come reato al momento della sua commissione ("caso Contrada", attinente, quindi, al principio della irretroattività in peius $)^{129}$. Esclusa la revocabilità della sentenza

${ }^{126}$ Cass., Sez. Un., 23 giugno 2016, n. 26259, cit., par. 6.2 motivazione. La tesi è del tutto in linea con la ricostruzione dottrinale richiamata alla nt. 112.

127 V., supra, par. 5.

${ }^{128}$ Cass., Sez. Un., 23 giugno 2016, n. 26259, cit., par. 7 motivazione. Criticamente, sul punto, LAVARINI, Barbara, I rimedi post iudicatum alla violazione dei canoni europei, cit., pp. 117-118.

129 Cass., Sez. I, 20 settembre 2017, n. 43112, caso in cui il ricorrente aveva interamente scontato la pena principale di dieci anni di reclusione irrogatagli dal Tribunale di Palermo con sentenza del 5 aprile 1996, confermata dalla Corte 
di condanna, la Suprema Corte ha individuato nel combinato disposto degli artt. 666 e 670 c.p.p. il rimedio invocabile per conformare l'ordinamento interno alla sentenza europea ai sensi dell'art. 46 CEDU. In conformità alla ricostruzione enunciata dalle Sezioni Unite nel "caso Gatto" ${ }^{130}$, la "sentenza Contrada" ha affermato che tali articoli «non possono che essere interpretati nel senso di consentire l'eliminazione degli effetti pregiudizievoli derivanti da una condanna emessa dal giudice italiano in violazione di una norma della Convenzione EDU, dovendosi ribadire che il garante della legalità della sentenza in fase esecutiva è il giudice dell'esecuzione, cui compete, se necessario, di ricondurre la decisione censurata ai canoni della legittimità» ${ }^{131}$.

Resta, comunque, da esplicitare la conclusione adottata dalla Corte, nel senso di dichiarare che la sentenza irrevocabile di condanna, a seguito della decisione emessa dalla Corte EDU, «non è suscettibile di ulteriore esecuzione e non è produttiva di ulteriori effetti penali» ${ }^{132}$. Si tratta di un rimedio attuativo del principio del nullum crimen: l'effetto, come nel caso della revoca per abolitio criminis, è che il condannato non potrà più essere punito, nè considerato colpevole per un fatto non previsto dalla legge come reato. Indubbiamente l'obiettivo perseguito dalla Suprema Corte in questa vicenda è stato perseguito forzando la lettera delle disposizioni processuali (in alternativa alla proposizione dell'incidente di costituzionalità), ma la conclusione è forse condivisibile quantomeno sulla scorta dell'interpretazione a fortiori ${ }^{133}$.

di appello di Palermo con sentenza del 25 febbraio 2006, per il reato di cui agli artt. 110, 416 e 416-bis c.p. Come è noto, la Corte EDU, con la decisione emessa il 14 aprile 2015, ha condannato lo Stato italiano per violazione dell'art. 7 CEDU, ritenendo che la fattispecie del concorso esterno nell'associazione di tipo mafioso fosse chiara e prevedibile solo a partire dal 1994, ovvero dal momento in cui interveniva la prima delle sentenze chiarificatrici delle Sezioni Unite in questa materia.

$130 \mathrm{~V}$., supra, par. 4.

${ }^{131}$ Cass., Sez. I, 20 settembre 2017, n. 43112, par. 5.5 motivazione.

${ }^{132}$ Cass., Sez. I, 20 settembre 2017, n. 43112, par. 5.5 motivazione.

${ }^{133}$ Si era infatti osservato, in dottrina, prima della pronuncia della Corte di cassazione nel "caso Contrada", che «a favore dell'interpretazione a fortiori dell'art. 673 c.p.p.» si sarebbe potuto invocare «la lettura estensiva» della 
Un analogo dubbio circa l'applicabilità o non dell'art. 673 c.p.p., in situazioni di acclarata violazione dei diritti fondamentali, concerne poi il caso della condanna per un fatto previsto da una norma incriminatrice interna che confligga con una norma di fonte UE a efficacia diretta ${ }^{134}$. Tenuto conto che la disapplicazione della norma nazionale incompatibile con il diritto $\mathrm{UE}^{135}$, pur non traducendosi in un fenomeno abrogativo, potrebbe essere ricondotta alla disciplina dell'art. 2 comma $2^{\circ}$ c.p., si è sostenuta l'operatività, in via analogica, dell'istituto previsto dall'art. 673 c.p.p., quale «"canale processuale” dell'intervento» in executivis già legittimato dalla legge penale sostanziale ${ }^{136}$.

disposizione fornita dalla "sentenza Mraidi", tanto più alla luce del già citato riferimento delle Sezioni Unite, in questa pronuncia, all'attivabilità del rimedio della revoca, non solo nei casi previsti dall'art. 2 comma $2^{\circ}$ c.p., ma anche in quelli di cui al primo comma (con la copertura dell'art. 25 comma $2^{\circ}$ Cost.). Così, LAVARINI, Barbara, I rimedi post iudicatum alla violazione dei canoni europei, cit., p. 118.

${ }^{134} \mathrm{Nel}$ senso che «in tutti i casi di incompatibilità tra norma penale e diritto dell'UE, se vi è stata sentenza definitiva di condanna per un fatto preveduto dalla norma penale inapplicabile, cessa l'esecuzione della condanna e ne vengono meno gli effetti penali», v. MARINUCCI, Giorgio; DOLCINI, Emilio, Manuale di Diritto Penale. Parte Generale, VI ediz., agg. a cura di Dolcini, Emilio; Gatta, Gian Luigi, Milano, Giuffrè, 2017, p. 53. V., in giurisprudenza, ad es., Cass., Sez. III, 3 giugno 2014, n. 30591, in C.E.D. Cass., rv. 259956.

${ }^{135}$ Ciò si è verificato con la "sentenza El Dridi" della Corte di Giustizia dell'UE, del 28 aprile 2011, causa C-61/11, che ha rilevato il contrasto del delitto di inottemperanza all'ordine di allontanamento intimato dal questore di cui all'art. 14 comma 5-ter T.U. immigrazione, con la direttiva rimpatri 2008/115/CE. V., in materia, ad es., MASERA, Luca; VIGANÒ, Francesco, Addio art. 14, in Diritto penale contemporaneo, 4 maggio 2011.

${ }^{136}$ Così, acutamente, LAVARINI, Barbara, I rimedi post iudicatum alla violazione dei canoni europei, cit., pp. 126-127, secondo la quale «non si tratta, infatti, di applicare per analogia un rimedio post iudicatum - operazione non consentita -, ma di applicare per analogia ad un rimedio post iudicatum, già previsto dalla legge nell'an, la disciplina processuale più adatta a calibrare il quomodo, il che, in una prospettiva di favor rei, appare accettabile» (corsivo nel testo). 


\section{BibliografiA}

BONTEMPELLI, Manfredi. La litispendenza penale, Milano, Giuffrè, 2017.

CANZIO, Giovanni. La giurisdizione e la esecuzione della pena, in Diritto penale contemporaneo, 26 aprile 2016. Disponibile in <https://www.penalecontemporaneo.it/>. Accesso: 7 settembre 2018.

CAPRIOLI, Francesco. Il principio del ne bis in idem, in CAPRIOLI, Francesco; VICOLI, Daniele, Procedura penale dell'esecuzione, II ediz., Torino, Giappichelli, 2011.

CAPRIOLI, Francesco. Giudicato e illegalità della pena: riflessioni a margine di una recente sentenza della Corte costituzionale, BARGIS, Marzia (a cura di), Studi in ricordo di Maria Gabriella Aimonetto, Milano, Giuffrè, 2013.

CAPRIOLI, Francesco. Il giudice e la legge processuale: il paradigma rovesciato, in L'Indice penale, 2017, pp. $967 \mathrm{~s}$.

CORDERO, Franco. Procedura penale, VII ediz., Milano, Giuffrè, 1983.

DE LUCA, Giuseppe. I limiti soggettivi della cosa giudicata penale, Milano, Giuffrè, 1963.

GAMBARDELLA, Marco. Annullamento di circostanze aggravanti incostituzionali e revoca parziale del giudicato di condanna, in Cassazione penale, 2012.

GAMBARDELLA, Marco. Overruling favorevole della Corte europea e revoca del giudicato di condanna: a proposito dei casi analoghi alla sentenza "Scoppola", in Cassazione penale, 2012.

LAMARQUE, Elisabetta; VIGANÒ, Francesco. Sulle ricadute interne della sentenza Scoppola, in Diritto penale contemporaneo, 31 marzo 2014.

LAVARINI, Barbara. L'esecutività della sentenza penale, Torino, Giappichelli, 2004.

LAVARINI, Barbara, I rimedi post iudicatum alla violazione dei canoni europei, in GAITO, Alfredo (a cura di), I princìpi europei del processo penale, Roma, Dike, 2016.

LEONE, Giovanni. Il mito del giudicato, in Scritti giuridici, vol. I, Napoli, Jovene, 1987.

LOZZI, Gilberto. Profili di una indagine sui rapporti tra «ne bis in idem» e concorso formale di reati, Milano, Giuffrè, 1974. 
LOZZI, Gilberto. Preclusioni, II) Diritto processuale penale (voce), in Enciclopedia giuridica Treccani, vol. XXVI, Roma, 1991.

MARINUCCI, Giorgio; DOLCINI, Emilio. Manuale di Diritto Penale. Parte Generale, VI ediz., agg. a cura di Dolcini, Emilio; GATtA, Gian Luigi, Milano, Giuffrè, 2017.

MASERA, Luca; VIGANÒ, Francesco. Addio art. 14, in Diritto penale contemporaneo, 4 maggio 2011.

MAZZA, Oliviero. Principio di legalità, diritto giurisprudenziale e giudice dell'esecuzione, in FIORIO, Carlo; FONTI, Rossella; MONTAGNA, Mariangela (a cura di), Inazione, controlli, esecuzione, Atti del Convegno in ricordo di Giovanni Dean, Pisa, Pacini Editore, 2017.

RAFARACI, Tommaso. Ne bis in idem (voce), in Enciclopedia del diritto, Annali, vol. III, Milano, Giuffrè, 2010.

RUGGERI, Stefano. Giudicato costituzionale, processo penale, diritti della persona, in Diritto penale contemporaneo - Rivista trimestrale, 2015, n. 1. Disponibile in <https://www.penalecontemporaneo.it/>. Accesso: 7 settembre 2018.

TROISI, Paolo. Flessibilità del giudicato penale e tutela dei diritti fondamentali, in Diritto penale contemporaneo, 2 aprile 2015. Disponibile in <https://www. penalecontemporaneo.it/>. Accesso: 7 settembre 2018.

VICOLI, Daniele. Il giudice dell'esecuzione, in CAPRIOLI, Francesco; VICOLI, Daniele, Procedura penale dell'esecuzione, II ediz., Torino, Giappichelli, 2011.

VICOLI, Daniele. L'illegittimità costituzionale della norma penale sanzionatoria travolge il giudicato: le nuove frontiere della fase esecutiva nei percorsi argomentativi delle Sezioni unite, in Rivista italiana di diritto e procedura penale, 2015.

VIGANÒ, Francesco. Pena illegittima e giudicato. Riflessioni in margine alla pronuncia delle Sezioni Unite che chiude la saga dei "fratelli minori" di Scoppola, in Diritto penale contemporaneo - Rivista trimestrale, 2014, n. 1. Disponibile in <https://www.penalecontemporaneo.it/>. Accesso: 7 settembre 2018.

VIGONI, Daniela. Giudicato ed esecuzione penale: confini normativi e frontiere giurisprudenziali, in Processo penale e giustizia, 2015, n. 4, pp. 1 s. Disponibile in <www.processopenaleegiustizia.it>. Accesso: 7 settembre 2018. 


\section{Informações adicionais e declarações dos autores (integridade científica)}

Declaração de conflito de interesses (conflict of interest declaration): o autor confirma que não há conflitos de interesse na realização das pesquisas expostas e na redação deste artigo.

Declaração de autoria e especificação das contribuições (declaration of authorship): todas e somente as pessoas que atendem os requisitos de autoria deste artigo estão listadas como autores.

Declaração de ineditismo e originalidade (declaration of originality): o autor assegura que o texto aqui publicado não foi divulgado anteriormente em outro meio e que futura republicação somente se realizará com a indicação expressa da referência desta publicação original; também atesta que não há plágio de terceiros ou autoplágio.

Dados do processo editorial

(http://www.ibraspp.com.br/revista/index.php/RBDPP/about/editorialPolicies)

- Recebido em: 20.07.2018

- Controle preliminar e verificação de plágio: 20.07.2018

- Avaliação 1: 02.08.2018

- Avaliação 2: 07.08.2018

- Decisão editorial preliminar: 21.07.2018

- Retorno rodada de correções 1: 07.09.2018

- Decisão editorial final: 22.09.2018
Equipe editorial envolvida

- Editor-chefe: 1 (VGV)

- Editores-associados: 2 (BC FC)

- Revisores: 2 


\section{COMO CITAR ESTE ARTIGO:}

BONTEMPELLI, Manfredi. La resistenza del giudicato alla violazione del principio di legalità penale. Revista Brasileira de Direito Processual Penal, Porto Alegre, vol. 4, n. 3, p. 1053-1094, set./dez. 2018.

https://doi.org/10.22197/rbdpp.v4i3.185

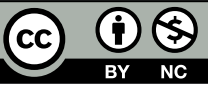

Esta obra está licenciada com uma Licença Creative Commons Atribuição-NãoComercial 4.0 Internacional. 